\title{
Discrete Hardy spaces
}

\author{
Paula Cerejeiras ${ }^{1}$, Uwe Kähler ${ }^{1}$, Min Kuํ ${ }^{1 \dagger}$, Frank Sommen ${ }^{2}$ \\ ${ }^{1}$ CIDMA, Department of Mathematics, University of Aveiro, Portugal \\ ${ }^{2}$ Clifford Research group, Department of Mathematical Analysis, Ghent University, Belgium
}

\begin{abstract}
We study the boundary behavior of discrete monogenic functions, i.e. null-solutions of a discrete Dirac operator, in the upper and lower half space. Calculating the Fourier symbol of the boundary operator we construct the corresponding discrete Hilbert transforms, the projection operators arising from them, and discuss the notion of discrete Hardy spaces. Hereby, we focus on the 3D-case with the generalization to the n-dimensional case being straightforward. Keywords: Discrete Cauchy
\end{abstract}

transform, Discrete monogenic functions, Clifford algebra

MSC(2000): primary: 44A15; secondary: 42A38, 42C40

\section{Introduction}

Hardy spaces are an important tool in harmonic analysis. Originally developed for problems in Complex Analysis they were extended in almost any conceivable way ( $[13,8,14])$. This is due to one principal fact: elements of a Hardy space can be identified with boundary values of analytic functions.

Particularly interesting for us here is the work of Li, McIntosh and Qian [14] which is quite detailed explained in [16]. Here the authors extend the concept of Hardy spaces via Fourier multipliers to the case of the Dirac operators in higher dimensions.

During the last ten years there is also a increased interest in obtaining discrete counterparts for continuous structures. Mainly driven by applications in physics one of these discrete counterparts is a discrete Dirac operator as a discrete equivalent to the continuous Dirac operator. In different contexts and by different methods such an operator was constructed in $[18,9,12,7]$, among others. Depending on their construction these discrete Dirac operators have different properties, even factorize different second order difference operators. As one would expect from

\footnotetext{
$0 \dagger$ Corresponding author

E-mail addresses: kumin0844@163.com, ukaehler@ua.pt, pceres@ua.pt, fs@cage.ugent.be

This work was supported by FEDER funds through COMPETE-Operational Programme Factors of Competitiveness ("Programa Operacional Factores de Competitividade"), by Portuguese fonds through the Center for Research and Development in Mathematics and Applications (University of Aveiro) and the Portuguese Foundation for Science and Technology ("FCT-Fundação para a Ciência e a Tecnologia"), within project PEstC/MAT/UI4106/2011 with COMPETE number FCOMP-01-0124-FEDER-022690 and by NNSF of China under Grant No.61170032. The first author is the recipient of Postdoctoral Foundation from FCT (Portugal) under Grant No.SFRH/BPD/74581/2010.
} 
the continuous case where the classic Dirac operator factorizes the Laplacian there is one particular operator which is more interesting from the practical point of view. In the case of a higher dimensional grid, i.e. $\mathbb{Z}^{n}$, it would be the star Laplacian. But here a major problem arises. Any factorization of the star Laplacian requires both forward and backward difference operators which in its turn requires a splitting of the usual Euclidean basis $[11,9, ?]$.

Nevertheless, first steps in the direction of a function theory for this kind of Dirac operator have been made with a general Cauchy formula, Fischer decomposition, and polynomials solutions (see $[10,1,4]$. But here resides another problem: the fundamental solution of the Dirac operator, the so-called discrete Cauchy kernel, is known only via a Fourier integral which severely limits its applicability from the analytic point of view. For instance, in $[5, ?, 10,6]$ the authors use the discrete fundamental solution to construct discrete boundary integral operators for a discrete version of the boundary element method similar to Ryabenkij [17]. Since the discrete fundamental solution is only known as a numerical approximation the results could be given only in the form that when the discrete integral equation over the boundary is solvable then the corresponding discrete potentials provide a solution to the corresponding difference equation. No direct characterization of the boundary values could be given. But that is what we are interested in: characterizations of null-solutions of the discrete Dirac operator based on its discrete boundary values, similar to the continuous case. This problem is not only affected by the expression of the fundamental solution, but also by the construction of the Dirac operator which involves forward and backward differences and also by the discrete boundary. In difference to the continuous case a discrete boundary contains three layers, two for the inner boundary and two for the outer boundary. This means that, for instance, a discrete Cauchy integral formula for the inner domain will require function values from both layers of inner boundary. A detailed exposition of this effect will be given in Section 3.

To obtain characterizations of the boundary values of discrete monogenic functions and the corresponding Hardy spaces we need to study the equation over the boundary. While in the discrete case there is no trace operator, i.e. a limit process to the boundary, the problem of having to use two layers for the boundary and the lack of an explicit closed expression of the Cauchy kernel makes such a study quite difficult. Nevertheless, we will show that it is possible to get the symbol of the boundary operator in Fourier domain. For an easier understanding we will restrict ourselves to the case of $d=3$ but the formulae and results are valid in any dimension.

The paper is organized as follows. In Section 2 we will recall some basic facts about discrete Dirac operators, the underlying algebraic structure, and its fundamental solution which will be needed in the sequel. In Section 3, we discuss discrete Bore-Pompeiu and Cauchy formulae and introduce the Cauchy transform. In this section which is essentially expository in its nature we also present the framework for discussing discrete boundary values. In Section 4 we finally discuss the question of characterization of discrete boundary values by studying the symbol of the operator which comes from the corresponding boundary equation. This allows us to get conditions for a function to be a boundary value of a discrete monogenic function in the upper and lower half plane. We will present these condition both in terms of the components and the function itself. Afterwards we get the corresponding Hilbert transforms (in the sense that they mimic the traditional Hilbert transform as an operator which squares to the identity). This allows us to get the projection operators (classically known as Plemelj or Hardy projections), Hardy spaces, and a decomposition theorem for functions on the boundary. 
In the last section we will show that we have indeed convergence for our formulae, i.e. when the lattice constant goes to zero we will recover the classic formulae from the continuous case.

\section{Preliminaries and notations}

Let us start with some basic facts of discrete function theory. Since we restrict ourselves to the case $d=3$ (without loss of generality) we consider the grid $\mathbb{Z}^{3}$ with orthonormal basis $e_{k}$, $k=1, \ldots, 3$. The standard forward and backward differences $\partial_{h}^{ \pm j}$ are given by

$$
\partial_{h}^{+j} f(m h)=h^{-1}\left(f\left(m h+e_{j} h\right)-f(m h)\right), \partial_{h}^{-j} f(m h)=h^{-1}\left(f(m h)-f\left(m h-e_{j} h\right)\right)
$$

where $h>0$ denotes the lattice constant.

As stated in the introduction we want to use a discrete Dirac operator which factorizes the Star-Laplacian $\Delta_{h}=\sum_{j=1}^{3} \partial_{h}^{+j} \partial_{h}^{-j}$. To get such an operator we follow the idea of $[1,7]$ by splitting each basis element $\mathrm{e}_{k}(k=1,2,3)$ into two basis elements $\mathrm{e}_{k}^{+}$and $\mathrm{e}_{k}^{-}(k=1,2,3)$, i.e., $\mathrm{e}_{k}=\mathrm{e}_{k}^{+}+\mathrm{e}_{k}^{-}(k=1,2,3)$ corresponding to the forward and backward directions. Let us remark that there is some freedom in choosing such a basis, special choices can be found in $[11,6,2]$. Here we choose the one satisfying the following relations:

$$
\left\{\begin{aligned}
e_{j}^{-} e_{k}^{-}+e_{k}^{-} e_{j}^{-} & =0 \\
e_{j}^{+} e_{k}^{+}+e_{k}^{+} e_{j}^{+} & =0 \\
e_{j}^{+} e_{k}^{-}+e_{k}^{-} e_{j}^{+} & =-\delta_{j k},
\end{aligned}\right.
$$

where $\delta_{j k}$ is the Kronecker delta. These basis elements generate a free algebra which is isomorphic to the Clifford algebra $\mathbb{C}_{3}$.

Furthermore, we consider functions defined on a subset $G$ of $\mathbb{Z}^{3}$ taking values in $\mathbb{C}_{3}$. Properties like $l_{p}$-summability $(1 \leqslant p<\infty)$ and so on, are defined for a $\mathbb{C}_{3}$-valued function by being ascribed to each component. The corresponding spaces are denoted, respectively, by $l_{p}\left(G, \mathbb{C}_{3}\right),(1 \leqslant p<+\infty)$ and so on.

The discrete Dirac operator is given by

$$
D_{h}^{+-}=\sum_{j=1}^{3} e_{j}^{+} \partial_{h}^{+j}+e_{j}^{-} \partial_{h}^{-j} .
$$

This operator factorizes the star-Laplacian $\Delta_{h}$, i.e.,

$$
\left(D_{h}^{+-}\right)^{2}=-\Delta_{h} \text { with } \Delta_{h}=: \sum_{j=1}^{m} \partial_{h}^{+j} \partial^{-j} .
$$

We will also need its adjoint operator, which is given by

$$
D_{h}^{-+}=\sum_{j=1}^{3} e_{j}^{+} \partial_{h}^{-j}+e_{j}^{-} \partial_{h}^{+j} .
$$

For more details we refer the reader to the literature, e.g. $[10,11,1,7,4]$. 


\subsection{Some remarks on the fundamental solution}

In this section we present a fundamental solution to the discrete Dirac operator and some basic facts which are required in the sequel.

Definition 2.1 A function $E_{h}^{-+}$defined on $\mathbb{Z}^{3}$ is called a discrete fundamental solution of $D_{h}^{-+}$ if it satisfies

$$
D_{h}^{-+} E_{h}^{-+}(m h)=\delta_{h}(m h), \forall m h \in G,
$$

where $\delta_{h}$ denotes the discrete Dirac function given by

$$
\delta_{h}(m h)=\left\{\begin{array}{cl}
h^{-3} & \text { if } \quad m h=0, \\
0 & \text { if } \quad m h \neq 0 .
\end{array}\right.
$$

There are several ways of constructing a fundamental solution. Here we will use the discrete Fourier transform on $l_{p}\left(\mathbb{Z}^{3}, \mathbb{C}_{3}\right)(1 \leqslant p<+\infty)$

$$
\mathcal{F}_{h} u(\xi)=\sum_{m \in \mathbb{Z}^{3}} e^{i<m h, \xi>} u(m h) h^{3}, \xi \in\left[-\frac{\pi}{h}, \frac{\pi}{h}\right]^{3},
$$

where $\left\langle x, \xi>=\sum_{j=1}^{3} x_{j} \xi_{j}\right.$ for arbitrary $x=\sum_{j=1}^{3} e_{j} x_{j}, \xi=\sum_{j=1}^{3} e_{j} \xi_{j} \in \mathbb{R}^{3}, x_{j}, \xi_{j} \in \mathbb{R}(j=1,2,3)$.

Its inverse is given by $\mathcal{F}_{h}^{-1}=R_{h} \mathcal{F}$ where $R_{h}$ denotes the restriction to the lattice $h \mathbb{Z}^{3}$ and $\mathcal{F}$ the (continuous) Fourier transform

$$
\mathcal{F} f(x)=\frac{1}{(2 \pi)^{3}} \int_{\mathbb{R}^{3}} e^{-i<x, \xi>} f(\xi) d \xi, \forall x \in \mathbb{R}^{3} .
$$

Let us remark that we have $\mathcal{F}_{h}\left(D_{h}^{-+} u\right)(\xi)=\left(\sum_{j=1}^{3} e_{j}^{+} \xi_{-j}^{D}+e_{j}^{-} \xi_{+j}^{D}\right) \mathcal{F}_{h} u(\xi)$ with $\xi_{ \pm j}^{D}=$ $\mp h^{-1}\left(1-e^{\mp i h \xi_{j}}\right)$ and $\mathcal{F}_{h}\left(\Delta_{h} u\right)(\xi)=\frac{4}{h^{2}} \sum_{j=1}^{3} \sin ^{2}\left(\frac{\xi_{j} h}{2}\right) \mathcal{F}_{h} u(\xi)$. Therefore, we can introduce the following denotations

$$
\widetilde{\xi}_{-}=\sum_{j=1}^{3} e_{j}^{+} \xi_{-j}^{D}+e_{j}^{-} \xi_{+j}^{D}
$$

and

$$
d^{2}=\frac{4}{h^{2}} \sum_{j=1}^{3} \sin ^{2}\left(\frac{\xi_{j} h}{2}\right)
$$

for the discrete Fourier symbol of $D_{h}^{-+}$and $\Delta$, respectively.

These observations allow us to obtain our fundamental solution as

$$
E_{h}^{-+}=R_{h} \mathcal{F}\left(\frac{\widetilde{\xi}_{-}}{d^{2}}\right)=\frac{1}{d^{2}} \sum_{j=1}^{3} e_{j}^{+} R_{h} \mathcal{F}\left(\xi_{-j}^{D}\right)+e_{j}^{-} R_{h} \mathcal{F}\left(\xi_{+j}^{D}\right) .
$$

Moreover, $\lim _{h \rightarrow 0} \frac{\widetilde{\xi}_{-}}{d^{2}}=\frac{-i \xi}{|\xi|^{2}}$ with $\xi=\sum_{j=1}^{3} e_{j} \xi_{j}$ and $|\xi|^{2}=\sum_{j=1}^{3} \xi_{j}^{2}, \xi_{j} \in \mathbb{R}(j=1,2,3)$. 
Lemma 2.2 For our fundamental solution $E^{-+}$we have

$$
\begin{aligned}
& \text { (i) } D_{h}^{-+} E_{h}^{-+}(m h)=\delta_{h}, \forall m h \in \mathbb{Z}^{3}, \\
& \text { (ii) } E_{h}^{-+} \in l_{p}\left(\mathbb{Z}^{3}, \mathbb{C}_{3}\right)(1 \leqslant p<+\infty) .
\end{aligned}
$$

Proof: Statement $(i)$ is obvious. (ii) follows directly from [11] where it was shown that for each component we have

$$
\left|\int_{[-\pi / h, \pi / h]^{3}} \frac{\xi_{ \pm j}^{D}}{d^{2}} e^{-i<x, \xi>} d \xi\right| \leqslant \frac{M h}{(|x|+h)^{3}}+\frac{M}{(|x|+h)^{2}}
$$

where $M>0$ is independent on $x \in h \mathbb{Z}^{3}$.

For the convergence of the discrete fundamental solution to the continuous one we have the following fundamental lemma.

Lemma 2.3 Let $E$ be the fundamental solution to the (continuous) Dirac operator in $\mathbb{R}^{3}$. For arbitrary $m \in \mathbb{Z}^{3}$ with $m \neq 0$, we have

$$
\left|E_{h}^{-+}(m h)-E(m h)\right| \leqslant C_{1} h /|m h|^{3},
$$

where $C_{1}>0$ is a constant independent on $h$.

Proof: Since the fundamental solution to Dirac operator in the the continuous case can be expressed via the Fourier transform as $E=\mathcal{F}\left(\frac{-i \xi}{|\xi|^{2}}\right)$ we have

$$
\begin{aligned}
& \left|E_{h}^{-+}(x)-E(x)\right|=\left|\frac{1}{(2 \pi)^{3}} \int_{\xi \in[-\pi / h, \pi / h]^{3}} \frac{\widetilde{\xi}_{-}}{d^{2}} e^{-i<x, \xi>} d \xi-\frac{1}{(2 \pi)^{3}} \int_{\mathbb{R}^{3}} \frac{-i \xi}{|\xi|^{2}} e^{-i<x, \xi>} d \xi\right| \\
& =\left|\int_{\xi \in[-\pi / h, \pi / h]^{3}} \frac{1}{(2 \pi)^{3}}\left(\frac{\widetilde{\xi}_{-}}{d^{2}}+\frac{i \xi}{|\xi|^{2}}\right) e^{-i<x, \xi>} d \xi+\int_{\xi \in \mathbb{R}^{3} \backslash[-\pi / h, \pi / h]^{3}} \frac{1}{(2 \pi)^{3}} \frac{i \xi}{|\xi|^{2}} e^{-i<x, \xi>} d \xi\right| .
\end{aligned}
$$

Now, noting that $\widetilde{\xi}_{-}=\sum_{j=1}^{3} e_{j}^{+} \xi_{-j}^{D}+e_{j}^{-} \xi_{+j}^{D}, e_{j}=e_{j}^{+}+e_{j}^{-}(j=1,2,3)$, for each component we can make the following estimate (using the Taylor expansions of $\cos h \xi_{j}, \sin h \xi_{j}$ and $d^{2} \leqslant|\xi|^{2}$, c.f. also reference [11])

$$
\begin{aligned}
& \left|\int_{\xi \in[-\pi / h, \pi / h]^{3}}\left(\frac{\xi_{ \pm j}^{D}}{d^{2}}+\frac{i \xi_{j}}{|\xi|^{2}}\right) e^{-i<x, \xi>} d \xi+\int_{\xi \in \mathbb{R}^{3} \backslash[-\pi / h, \pi / h]^{3}} \frac{i \xi_{j}}{|\xi|^{2}} e^{-i<x, \xi>} d \xi\right| \\
& =\mid \int_{\xi \in[-\pi / h, \pi / h]^{3}} \mp \frac{1-\cos h \xi_{j}}{d^{2} h} e^{-i<x, \xi>} d \xi+i \int_{\xi \in[-\pi / h, \pi / h]^{3}}\left(\frac{\xi_{j}}{|\xi|^{2}}-\frac{\sin h \xi_{j}}{d^{2} h}\right) e^{-i<x, \xi>} d \xi \\
& +i \int_{\xi \in\left\{\mathbb{R}^{3} \backslash[-\pi / h, \pi / h]^{3}\right\}} \frac{\xi_{j}}{|\xi|^{2}} e^{-i<x, \xi>} d \xi \mid \\
& \leqslant\left|\int_{\xi \in[-\pi / h, \pi / h]^{3}} \frac{1-\cos h \xi_{j}}{d^{2} h} e^{-i<x, \xi>} d \xi\right|+\mid \int_{\xi \in[-h / \pi, h / \pi]^{3}}\left(\frac{\sin h \xi_{j}}{d^{2} h}-\frac{\xi_{j}}{|\xi|^{2}}\right) e^{-i<x, \xi>} d \xi \\
& -\int_{\xi \in \mathbb{R}^{3} \backslash[-\pi / h, \pi / h]^{3}} \frac{\xi_{j}}{|\xi|^{2}} e^{-i<x, \xi>} d \xi \mid \leqslant C_{2} \frac{h}{|x|^{3}}, x \neq 0,
\end{aligned}
$$


with $C_{2}>0$ being a constant independent on $h$. Hence we get

$$
\left|E_{h}^{-+}(x)-E(x)\right| \leqslant \frac{C_{1} h}{|x|^{3}}, x \neq 0
$$

Therefore, it follows that

$$
\left|E_{h}^{-+}(m h)-E(m h)\right| \leqslant C_{1} h /|m h|^{3}, \forall m \in \mathbb{Z}^{3}, m \neq 0,
$$

where $C_{1}>0$ is a constant independent on $h$.

Remark 2.4 In the same way we can also obtain fundamental solutions to other discrete operators such as $D_{h}^{+-}$, where we get $E_{h}^{+-}=R_{h} \mathcal{F}\left(\frac{\xi_{-}}{d^{2}}\right)=\frac{1}{d^{2}} \sum_{j=1}^{3} e_{j}^{+} R_{h} \mathcal{F}\left(\xi_{+j}^{D}\right)+e_{j}^{-} R_{h} \mathcal{F}\left(\xi_{-j}^{D}\right)$ with $\xi_{-}=\sum_{j=1}^{3} e_{j}^{+} \xi_{+j}^{D}+e_{j}^{-} \xi_{-j}^{D}$ and $\xi_{ \pm j}^{D}=\mp h^{-1}\left(1-e^{\mp i h \xi_{j}}\right)$. The convergence is similar to Lemma 2.3.

\subsection{Discrete Stokes formula for $\mathbb{Z}^{3}$ and $\mathbb{Z}_{+}^{3}$}

We also need the discrete Stokes formula with respect to our Dirac operator for functions defined on $\mathbb{Z}^{3}$ and $\mathbb{Z}_{+}^{3}$, respectively. To this end we denote by $n=\left(\underline{n}, n_{3}\right) \in \mathbb{Z}^{3}$ a point ofon the grid with $\underline{n}=\left(n_{1}, n_{2}\right) \in \mathbb{Z}^{2}$. For the upper half space $\mathbb{Z}_{+}^{3}$ we have $n_{3}>0$. Furthermore, in cases where $h$ is fixed (except in the last section) we abbreviate $f(n):=f(h n), n \in \mathbb{Z}^{3}$. In the case where changes affect only the $j^{t h}$-coordinate we write $f\left(n_{j}\right):=f\left(h n_{1}, \ldots, h n_{j}, \ldots, h n_{3}\right)$ where it can be done without arising confusion.

Theorem 2.5 Consider our operators $D_{h}^{+-}$and $D_{h}^{-+}$. Let $f, g \in l_{p}\left(\mathbb{Z}^{3}, \mathbb{C}_{3}\right)$ with $1 \leqslant p<+\infty$ then we have

$$
-\sum_{n \in \mathbb{Z}^{3}}\left[g(n) D_{h}^{-+}\right] f(n) h^{3}=\sum_{n \in \mathbb{Z}^{3}} g(n)\left[D_{h}^{+-} f(n)\right] h^{3} .
$$


Proof: Using the definition of $D_{h}^{+-}$and $D_{h}^{-+}$we get

$$
\begin{aligned}
& -\sum_{n \in \mathbb{Z}^{3}}\left[g(n) D_{h}^{-}\right] f(n) h^{3}=-\sum_{\left(\underline{n}, n_{3}\right) \in \mathbb{Z}^{3}} \sum_{j=1}^{3}\left[\partial^{+j} g\left(n_{j}\right) e_{j}^{-}+\partial^{-j} g\left(n_{j}\right) e_{j}^{+}\right] f\left(n_{j}\right) h^{3} \\
= & -\sum_{\left(\underline{n}, n_{3}\right) \in \mathbb{Z}^{3}} \sum_{j=1}^{3}\left\{\left[g\left(n_{j}+1\right)-g\left(n_{j}\right)\right] e_{j}^{-}+\left[g\left(n_{j}\right)-g\left(n_{j}-1\right)\right] e_{j}^{+}\right\} f\left(n_{j}\right) h^{3} \\
= & -\sum_{\left(\underline{n}, n_{3}\right) \in \mathbb{Z}^{3}} \sum_{j=1}^{3}\left[g\left(n_{j}+1\right) e_{j}^{-} f\left(n_{j}\right)-g\left(n_{j}\right) e_{j}^{-} f\left(n_{j}\right)+g\left(n_{j}\right) e_{j}^{+} f\left(n_{j}\right)-g\left(n_{j}-1\right) e_{j}^{+} f\left(n_{j}\right)\right] h^{3} \\
= & -\sum_{\left(\underline{n}, n_{3}\right) \in \mathbb{Z}^{3}} \sum_{j=1}^{3}\left[g\left(n_{j}\right) e_{j}^{-} f\left(n_{j}-1\right)-g\left(n_{j}\right) e_{j}^{-} f\left(n_{j}\right)+g\left(n_{j}\right) e_{j}^{+} f\left(n_{j}\right)-g\left(n_{j}\right) e_{j}^{+} f\left(n_{j}+1\right)\right] h^{3} \\
= & \sum_{\left(\underline{n}, n_{3}\right) \in \mathbb{Z}^{3}} \sum_{j=1}^{3}\left[g\left(n_{j}\right) e_{j}^{-} f\left(n_{j}\right)-g\left(n_{j}\right) e_{j}^{-} f\left(n_{j}-1\right)+g\left(n_{j}\right) e_{j}^{+} f\left(n_{j}+1\right)-g\left(n_{j}\right) e_{j}^{+} f\left(n_{j}\right)\right] h^{3} \\
= & \sum_{\left(\underline{n}, n_{3}\right) \in \mathbb{Z}^{3}} \sum_{j=1}^{3} g\left(n_{j}\right)\left[e_{j}^{-} \partial^{-j} f\left(n_{j}\right)+e_{j}^{+} \partial^{+j} f\left(n_{j}\right)\right]=\sum_{n \in \mathbb{Z}^{3}} g(n)\left[D_{h}^{+} f(n)\right] h^{3} .
\end{aligned}
$$

For the special case where $g(n)=E_{h}^{-+}(n-m)$ is a translate of the fundamental solution to operator $D_{h}^{-+}$with $m \in \mathbb{Z}^{3}$ fixed we get the following corollary.

Corollary 2.6 Let $f \in l_{p}\left(\mathbb{Z}^{3}, \mathbb{C}_{3}\right)$ with $1 \leqslant p<+\infty$ then for arbitrary $m \in \mathbb{Z}^{3}$ we have

$$
f(m)=-\sum_{n \in \mathbb{Z}^{3}} E_{h}^{-+}(n-m)\left[D_{h}^{+-} f(n)\right] h^{3} .
$$

Furthermore, as a simple consequence of the above corollary we get the following discrete unique continuation principle.

Corollary 2.7 If $f \in l_{p}\left(\mathbb{Z}^{3}, \mathbb{C}_{3}\right)(1 \leqslant p<+\infty)$ is left monogenic with respect to operator $D_{h}^{+-}$, i.e., $D_{h}^{+-} f=0, \forall m \in \mathbb{Z}^{3}$, then

$$
f(m)=0, m \in \mathbb{Z}^{3} .
$$

Let us now consider the case of the upper half space $\left(\underline{n}, n_{3}\right) \in \mathbb{Z}^{2} \times \mathbb{Z}_{+}$. Here, we get the following theorem.

Theorem 2.8 For $f, g \in l_{p}\left(\mathbb{Z}^{2} \times \mathbb{Z}_{+}, \mathbb{C}_{3}\right)(1 \leqslant p<+\infty)$ we have

$$
\begin{aligned}
-\sum_{\left(\underline{n}, n_{3}\right) \in \mathbb{Z}^{2} \times \mathbb{Z}_{+}}\left[g(n) D_{h}^{-+}\right] f(n) h^{3} & =\sum_{\left(\underline{n}, n_{3}\right) \in \mathbb{Z}^{2} \times \mathbb{Z}_{+}} g(n)\left[D_{h}^{+-} f(n)\right] h^{3} \\
& +\sum_{\underline{n} \in \mathbb{Z}^{2}}\left[g(\underline{n}, 0) e_{3}^{+} f(\underline{n}, 1)+g(\underline{n}, 1) e_{3}^{-} f(\underline{n}, 0)\right] h^{3} .
\end{aligned}
$$


Proof: Starting again from the definition of our operators $D_{h}^{-+}$and $D_{h}^{+-}$we obtain

$$
\begin{aligned}
- & \sum_{\left(\underline{n}, n_{3}\right) \in \mathbb{Z}^{2} \times \mathbb{Z}_{+}}\left[g(n) D_{h}^{-+}\right] f(n) h^{3} \\
= & -\sum_{\left(\underline{n}, n_{3}\right) \in \mathbb{Z}^{2} \times \mathbb{Z}_{+}} \sum_{j=1}^{2}\left[\partial^{+j} g\left(n_{j}\right) e_{j}^{-}+\partial^{-j} g\left(n_{j}\right) e_{j}^{+}\right] f\left(n_{j}\right) h^{3} \\
- & \sum_{\left(\underline{n}, n_{3}\right) \in \mathbb{Z}^{2} \times \mathbb{Z}_{+}}\left[\partial^{+3} g\left(n_{3}\right) e_{3}^{-}+\partial^{-3} g\left(n_{3}\right) e_{3}^{+}\right] f\left(n_{3}\right) h^{3} \\
= & \sum_{\left(\underline{n}, n_{3}\right) \in \mathbb{Z}^{2}} \sum_{j=1}^{2} g\left(n_{j}\right)\left[e_{j}^{-} \partial^{-j} f\left(n_{j}\right)+e_{j}^{+} \partial^{+j} f\left(n_{j}\right)\right] h^{3} \\
- & \sum_{\underline{n} \in \mathbb{Z}^{2}}\left\{\sum_{n_{3} \geqslant 1}\left[\left(g\left(n_{3}+1\right)-g\left(n_{3}\right)\right) e_{3}^{-} f\left(n_{3}\right)+\left(g\left(n_{3}\right)-g\left(n_{3}-1\right)\right) e_{3}^{+} f\left(n_{3}\right)\right] h^{3}\right\} \\
= & \sum_{\left(\underline{n}, n_{3}\right) \in \mathbb{Z}^{2} \times \mathbb{Z}_{+}} \sum_{j=1}^{2} g\left(n_{j}\right)\left[e_{j}^{-} \partial^{-j} f\left(n_{j}\right)+e_{j}^{+} \partial^{+j} f\left(n_{j}\right)\right] h^{3} \\
- & \sum_{\underline{n} \in \mathbb{Z}^{2}}\left\{\sum_{n_{3} \geqslant 1}\left[g\left(n_{3}+1\right) e_{3}^{-} f\left(n_{3}\right)-g\left(n_{3}\right) e_{3}^{-} f\left(n_{3}\right)+g\left(n_{3}\right) e_{3}^{+} f\left(n_{3}\right)-g\left(n_{3}-1\right) e_{3}^{+} f\left(n_{3}\right)\right]\right\} h^{3} .
\end{aligned}
$$

For the last term of the above equality, we get

$$
\begin{aligned}
& -\sum_{n_{3} \geqslant 1}\left[g\left(n_{3}+1\right) e_{3}^{-} f\left(n_{3}\right)-g\left(n_{3}\right) e_{3}^{-} f\left(n_{3}\right)+g\left(n_{3}\right) e_{3}^{+} f\left(n_{3}\right)-g\left(n_{3}-1\right) e_{3}^{+} f\left(n_{3}\right)\right] \\
= & -\sum_{n_{3} \geqslant 2} g\left(n_{3}\right) e_{3}^{-} f\left(n_{3}-1\right)+\sum_{n_{3} \geqslant 1} g\left(n_{3}\right) e_{3}^{-} f\left(n_{3}\right)-\sum_{n_{3} \geqslant 1} g\left(n_{3}\right) e_{3}^{+} f\left(n_{3}\right)+\sum_{n_{3} \geqslant 0} g\left(n_{3}\right) e_{3}^{+} f\left(n_{3}+1\right) \\
= & \sum_{n_{3} \geqslant 1} g\left(n_{3}\right) e_{3}^{-}\left[f\left(n_{3}\right)-f\left(n_{3}-1\right)\right]+\sum_{n_{3} \geqslant 1} g\left(n_{3}\right) e_{3}^{+}\left[f\left(n_{3}+1\right)-f\left(n_{3}\right)\right]+g(0) e_{3}^{+} f(1)+g(1) e_{3}^{-} f(0) \\
= & \sum_{n_{3} \geqslant 1} g\left(n_{3}\right)\left[e_{3}^{-} \partial^{-3} f\left(n_{3}\right)+e_{3}^{+} \partial^{+3} f\left(n_{3}\right)\right]+g(0) e_{3}^{+} f(1)+g(1) e_{3}^{-} f(0) .
\end{aligned}
$$

Such that one has

$$
\begin{aligned}
-\sum_{\left(\underline{n}, n_{3}\right) \in \mathbb{Z}^{2} \times \mathbb{Z}_{+}}\left[g(n) D_{h}^{-}\right] f(n) h^{3} & =\sum_{\left(\underline{n}, n_{3}\right) \in \mathbb{Z}^{2} \times \mathbb{Z}_{+}} g(n)\left[D_{h}^{+} f(n)\right] h^{3} \\
& +\sum_{\underline{n} \in \mathbb{Z}^{2}}\left[g(\underline{n}, 0) e_{3}^{+} f(\underline{n}, 1)+g(\underline{n}, 1) e_{3}^{-} f(\underline{n}, 0)\right] h^{3} .
\end{aligned}
$$


In the same way we get for the lower half space $\left(\underline{n}, n_{3}\right) \in \mathbb{Z}^{2} \times \mathbb{Z}_{-}$the following theorem.

$$
\begin{aligned}
- & \sum_{\left(\underline{n}, n_{3}\right) \in \mathbb{Z}^{2} \times \mathbb{Z}_{-}}\left[g(n) D_{h}^{-+}\right] f(n) h^{3}=\sum_{\left(\underline{n}, n_{3}\right) \in \mathbb{Z}^{2} \times \mathbb{Z}_{-}} \sum_{j=1}^{2} g\left(n_{j}\right)\left[e_{j}^{-} \partial^{-j} f\left(n_{j}\right)+e_{j}^{+} \partial^{+j} f\left(n_{j}\right)\right] h^{3} \\
- & \sum_{\underline{n} \in \mathbb{Z}^{2}}\left\{\sum_{n_{3} \leqslant-1}\left[g\left(n_{3}+1\right) e_{3}^{-} f\left(n_{3}\right)-g\left(n_{3}\right) e_{3}^{-} f\left(n_{3}\right)+g\left(n_{3}\right) e_{3}^{+} f\left(n_{3}\right)+g\left(n_{3}-1\right) e_{3}^{+} f\left(n_{3}\right)\right]\right\} h^{3} \\
= & \sum_{\left(\underline{n}, n_{3}\right) \in \mathbb{Z}^{2} \times \mathbb{Z}_{-}} \sum_{j=1}^{2} g\left(n_{j}\right)\left[e_{j}^{-} \partial^{-j} f\left(n_{j}\right)+e_{j}^{+} \partial^{+j} f\left(n_{j}\right)\right] h^{3} \\
& \sum_{\underline{n} \in \mathbb{Z}^{2}}\left\{-\sum_{n_{3} \leqslant 0} g\left(n_{3}\right) e_{3}^{-} f\left(n_{3}-1\right)+\sum_{n_{3} \leqslant-1} g\left(n_{3}\right) e_{3}^{-} f\left(n_{3}\right)-\sum_{n_{3} \leqslant-1} g\left(n_{3}\right) e_{3}^{+} f\left(n_{3}\right)+\sum_{n_{3} \leqslant-2} g\left(n_{3}\right) e_{3}^{+} f\left(n_{3}+1\right)\right\} h^{3} \\
= & \sum_{\left(\underline{n}, n_{3}\right) \in \mathbb{Z}^{2} \times \mathbb{Z}_{-}} \sum_{j=1}^{2} g\left(n_{j}\right)\left[e_{j}^{-} \partial^{-j} f\left(n_{j}\right)+e_{j}^{+} \partial^{+j} f\left(n_{j}\right)\right] h^{3}+\sum_{\underline{n} \in \mathbb{Z}^{2}} \sum_{n_{3} \leqslant-1} g\left(n_{3}\right)\left[e_{3}^{-} \partial^{-3} f\left(n_{3}\right)+e_{3}^{+} \partial^{+3} f\left(n_{3}\right)\right] h^{3} \\
- & \sum_{\underline{n} \in \mathbb{Z}^{2}}\left[g(\underline{n}, 0) e_{3}^{-} f(\underline{n},-1)+g(\underline{n},-1) e_{3}^{+} f(\underline{n}, 0)\right] h^{3} .
\end{aligned}
$$

Theorem 2.9 For $f, g \in l_{p}\left(\mathbb{Z}^{2} \times \mathbb{Z}_{-}, \mathbb{C}_{3}\right)(1 \leqslant p<+\infty)$ we have

$$
\begin{aligned}
& -\sum_{\left(\underline{n}, n_{3}\right) \in \mathbb{Z}^{2} \times \mathbb{Z}_{-}}\left[g(n) D_{h}^{-+}\right] f(n) h^{3}=\sum_{\left(\underline{n}, n_{3}\right) \in \mathbb{Z}^{2} \times \mathbb{Z}_{-}} g(n)\left[D_{h}^{+-} f(n)\right] h^{3} \\
& -\sum_{\underline{n} \in \mathbb{Z}^{2}}\left[g(\underline{n}, 0) e_{3}^{-} f(\underline{n},-1)+g(\underline{n},-1) e_{3}^{+} f(\underline{n}, 0)\right] h^{3} .
\end{aligned}
$$

\section{Borel-Pompeiu and Cauchy formula in the case of $\mathbb{Z}_{+}^{3}$}

As usual by combining the Stokes formula of the previous section with translates of the fundamental solution we will obtain the Borel-Pompeiu and Cauchy formulae on the upper and lower half lattices of $\mathbb{Z}_{ \pm}^{3}$. This will be used to define discrete Cauchy transforms on the upper and lower half lattices of $\mathbb{Z}_{ \pm}^{3}$.

Let us start with the Borel-Pompeiu formula.

Theorem 3.1 Let $E_{h}^{-+}$be the discrete fundamental solution to operator $D_{h}^{-+}$, and function $f \in l_{p}\left(\mathbb{Z}^{2} \times \mathbb{Z}_{+}, \mathbb{C}_{3}\right), 1 \leqslant p<+\infty$, then we have

$$
\begin{aligned}
& \sum_{\underline{n} \in \mathbb{Z}^{2}}\left[E_{h}^{-+}\left(\underline{n}-\underline{m},-m_{3}\right) e_{3}^{+} f(\underline{n}, 1)+E_{h}^{-+}\left(\underline{n}-\underline{m}, 1-m_{3}\right) e_{3}^{-} f(\underline{n}, 0)\right] h^{3} \\
& +\sum_{\left(\underline{n}, n_{3}\right) \in \mathbb{Z}^{2} \times \mathbb{Z}_{+}} E_{h}^{-+}(n-m)\left[D_{h}^{+-} f(n)\right] h^{3}=\left\{\begin{array}{cl}
0, & \text { if } m=\left(\underline{m}, m_{3}\right) \notin \mathbb{Z}^{2} \times \mathbb{Z}_{+}, \\
-f(m), & \text { if } m=\left(\underline{m}, m_{3}\right) \in \mathbb{Z}^{2} \times \mathbb{Z}_{+} .
\end{array}\right.
\end{aligned}
$$

Proof: For arbitrary $m=\left(\underline{m}, m_{3}\right) \in \mathbb{Z}^{3}$, substituting $g=E_{h}^{-+}(\cdot-m)$ in our discrete Stokes 
formula for the upper half space we obtain the discrete Borel-Pompeiu formula as follows

$$
\begin{aligned}
& -\sum_{\left(\underline{n}, n_{3}\right) \in \mathbb{Z}^{2} \times \mathbb{Z}_{+}}\left[E_{h}^{-+}(n-m) D_{h}^{-+}\right] f(n) h^{3}=\sum_{\left(\underline{n}, n_{3}\right) \in \mathbb{Z}^{2} \times \mathbb{Z}_{+}} E_{h}^{-+}(n-m)\left[D_{h}^{+-} f(n)\right] h^{3} \\
& +\sum_{\underline{n} \in \mathbb{Z}^{2}}\left[E_{h}^{-+}\left(\underline{n}-\underline{m},-m_{3}\right) e_{3}^{+} f(\underline{n}, 1)+E_{h}^{-+}\left(\underline{n}-\underline{m}, 1-m_{3}\right) e_{3}^{-} f(\underline{n}, 0)\right] h^{3}
\end{aligned}
$$

which leads to

$$
\begin{aligned}
& \sum_{\underline{n} \in \mathbb{Z}^{2}}\left[E_{h}^{-+}\left(\underline{n}-\underline{m},-m_{3}\right) e_{3}^{+} f(\underline{n}, 1)+E_{h}^{-+}\left(\underline{n}-\underline{m}, 1-m_{3}\right) e_{3}^{-} f(\underline{n}, 0)\right] h^{3} \\
& +\sum_{\left(\underline{n}, n_{3}\right) \in \mathbb{Z}^{2} \times \mathbb{Z}_{+}} E_{h}^{-+}(n-m)\left[D_{h}^{+-} f(n)\right] h^{3}=\left\{\begin{array}{cl}
0, & \text { if } m=\left(\underline{m}, m_{3}\right) \notin \mathbb{Z}^{2} \times \mathbb{Z}_{+}, \\
-f(m), & \text { if } m=\left(\underline{m}, m_{3}\right) \in \mathbb{Z}^{2} \times \mathbb{Z}_{+} .
\end{array}\right.
\end{aligned}
$$

Remark 3.2 In fact there are several ways to construct a Borel-Pompeiu formula. The simplest and at the same time the one which requires the most work is by direct calculation. Another approach which uses the characteristic function appears in [1]. The final form of the BorelPompeiu formula depends also on the definition of the interior and the boundary of the domain.

Now, as a special case we obtain a discrete Cauchy formula.

Theorem 3.3 Let $f \in l_{p}\left(\mathbb{Z}^{2} \times \mathbb{Z}_{+}, \mathbb{C}_{3}\right), 1 \leqslant p<+\infty$, be a discrete left monogenic function with respect to operator $D_{h}^{+-}$, then it holds the upper discrete Cauchy formula

$$
\begin{array}{r}
\sum_{\underline{n} \in \mathbb{Z}^{2}}\left[E_{h}^{-+}\left(\underline{n}-\underline{m},-m_{3}\right) e_{3}^{+} f(\underline{n}, 1)+E_{h}^{-+}\left(\underline{n}-\underline{m}, 1-m_{3}\right) e_{3}^{-} f(\underline{n}, 0)\right] h^{3} \\
=\left\{\begin{array}{cc}
0, & \text { if } m_{3} \leqslant 0, \\
-f(m), & \text { if } m_{3}>0 .
\end{array}\right.
\end{array}
$$

In the same way the lower discrete Cauchy formula can also be given by

$$
\begin{array}{r}
\sum_{\underline{n} \in \mathbb{Z}^{2}}\left[E_{h}^{-+}\left(\underline{n}-\underline{m},-1-m_{3}\right) e_{3}^{+} f(\underline{n}, 0)+E_{h}^{-+}\left(\underline{n}-\underline{m},-m_{3}\right) e_{3}^{-} f(\underline{n},-1)\right] h^{3} \\
=\left\{\begin{array}{cc}
0, & \text { if } m_{3} \geqslant 0, \\
f(m), & \text { if } m_{3}<0 .
\end{array}\right.
\end{array}
$$

These Cauchy formulae allow us to introduce the following discrete Cauchy transforms.

Definition 3.4 For a discrete $l_{p}$-function $f, 1 \leqslant p<+\infty$, defined on the boundary layers $(\underline{n}, 0),(\underline{n}, 1)$ with $\underline{n} \in \mathbb{Z}^{2}$, we define the upper Cauchy transform for $m=\left(\underline{m}, m_{3}\right)=$ $\left(m_{1}, m_{2}, m_{3}\right) \in \mathbb{Z}^{3} \times \mathbb{Z}_{+}$as

$$
C^{+}[f](m)=-\sum_{\underline{n} \in \mathbb{Z}^{2}}\left[E_{h}^{-+}\left(\underline{n}-\underline{m},-m_{3}\right) e_{3}^{+} f(\underline{n}, 1)+E_{h}^{-+}\left(\underline{n}-\underline{m}, 1-m_{3}\right) e_{3}^{-} f(\underline{n}, 0)\right] h^{3},
$$


and for a discrete $l_{p}$-function $f, 1 \leqslant p<\infty$, defined on the boundary layers $(\underline{n},-1),(\underline{n}, 0)$ with $\underline{n} \in \mathbb{Z}^{2}$, we define the lower Cauchy transform at $m=\left(\underline{m}, m_{3}\right)=\left(m_{1}, m_{2}, m_{3}\right) \in \mathbb{Z}^{3} \times \mathbb{Z}_{-}$by

$$
C^{-}[f](m)=\sum_{\underline{n} \in \mathbb{Z}^{2}}\left[E_{h}^{-+}\left(\underline{n}-\underline{m},-1-m_{3}\right) e_{3}^{+} f(\underline{n}, 0)+E_{h}^{-+}\left(\underline{n}-\underline{m},-m_{3}\right) e_{3}^{-} f(\underline{n},-1)\right] h^{3} .
$$

From our discrete Cauchy formulae (3.13) and (3.14) we can see the dependence of these transforms on three different layers (two for each).

By applying Hölder's inequality and the properties of the fundamental solution to operator $D_{h}^{-+}$we get the following theorem.

Theorem 3.5 Consider the upper and lower Cauchy transforms as given by (3.15) and (3.16), respectively. Then we have

$$
\begin{aligned}
& \text { (i) } C^{+}[f] \in l_{p}\left(\mathbb{Z}_{+}^{3}, \mathbb{C}_{3}\right), C^{-}[f] \in l_{p}\left(\mathbb{Z}_{-}^{3}, \mathbb{C}_{3}\right)(1 \leqslant p<+\infty), \\
& \text { (ii) } D_{h}^{+-} C^{+}[f](m)=0, \forall m=\left(\underline{m}, m_{3}\right) \in \mathbb{Z}^{3} \text { with } m_{3}>1, \\
& \text { (iii) } D_{h}^{+-} C^{-}[f](m)=0, \forall m=\left(\underline{m}, m_{3}\right) \in \mathbb{Z}^{3} \text { with } m_{3}<-1 .
\end{aligned}
$$

Proof: We only prove (ii) since the proof of $(i i i)$ is similar. By denoting $D_{m}^{+-}$the Dirac operator $D^{+-}$acting on $m$ we have for $m_{3}>1$

$$
\begin{aligned}
D_{m}^{+-} C^{+}[f](m)= & -\sum_{\underline{n} \in \mathbb{Z}^{2}}\left[D_{m}^{+-} E_{h}^{-+}\left(\underline{n}-\underline{m},-m_{3}\right) e_{3}^{+} f(\underline{n}, 1)\right. \\
& \left.+D_{m}^{+-} E_{h}^{-+}\left(\underline{n}-\underline{m}, 1-m_{3}\right) e_{3}^{-} f(\underline{n}, 0)\right] h^{3} \\
= & \sum_{\underline{n} \in \mathbb{Z}^{2}}\left[D_{-m}^{-+} E_{h}^{-+}\left(\underline{n}-\underline{m},-m_{3}\right) e_{3}^{+} f(\underline{n}, 1)\right. \\
& \left.+D_{-m}^{-+} E_{h}^{-+}\left(\underline{n}-\underline{m}, 1-m_{3}\right) e_{3}^{-} f(\underline{n}, 0)\right] h^{3}=0 .
\end{aligned}
$$

For establishing a global discrete Cauchy formula we have to solve several problems. First of all, we introduce the following function space

$$
\mathfrak{F}\left(\mathbb{Z}^{3}\right)=\left\{f: \mathbb{Z}^{3} \rightarrow \mathbb{C}_{3} \mid f=f^{+}+f^{-}\right\}
$$

where functions $f^{+}, f^{-}$satisfy

$$
f^{+}(m)=0 \text { if } m_{3}<0 \text {, and } D_{h}^{+-} f^{+}(m)=0 \text { if } m_{3}>0 \text { and } D_{h}^{+-} f^{+}(\underline{m},-1)=0,
$$

and

$$
f^{-}(m)=0 \text { if } m_{3}>0, \text { and } D_{h}^{+-} f^{-}(m)=0 \text { if } m_{3}<0 \text { and } D_{h}^{+-} f^{-}(\underline{m}, 1)=0 .
$$

This implies that

$$
f(m)= \begin{cases}f^{+}(m), & m_{3}>0 \\ f^{+}(\underline{m}, 0)+f^{-}(\underline{m}, 0), & m_{3}=0 \\ f^{-}(m), & m_{3}<0\end{cases}
$$


while $D_{h}^{+-} f(m)=0$ whenever $m_{3} \neq 0$. We would like to point out that no conditions of $f$ on the 0-layer will be imposed. Furthermore, at the moment we also do not discuss if this decomposition exists for all $l_{p}$-functions or if it is unique.

This idea of the decomposition results in the following theorem.

Theorem 3.6 Let function $f \in l_{p}\left(\mathbb{Z}^{3}, \mathbb{C}_{3}\right), 1 \leqslant p<+\infty$, be a global discrete function, which can be decomposed into $f=f^{+}+f^{-}$where

$$
f^{+}(m)=0 \text { if } m_{3}<0 \text {, and } D_{h}^{+-} f^{+}(m)=0 \text { if } m_{3}>0 \text { or } m_{3}=-1,
$$

and

$$
f^{-}(m)=0 \text { if } m_{3}>0 \text {, and } D_{h}^{+-} f^{-}(m)=0 \text { if } m_{3}<0 \text { or } m_{3}=1 .
$$

Then function $f$ is left discrete monogenic with respect to operator $D_{h}^{+-}$for $m \in \mathbb{Z}^{3}$ with $m_{3} \neq 0$ and its discrete Cauchy transform is given by

$$
C[f](m)=C^{+}\left[f^{+}\right](m)+C^{-}\left[f^{-}\right](m), m \in \mathbb{Z}^{3} .
$$

Proof: Applying the upper and lower Cauchy formulas (3.13) and (3.14), for arbitrary $m \in \mathbb{Z}^{3}$ such that $m_{3} \neq-1,0,+1$, we have

$$
f(m)=\left(C^{+}+C^{-}\right)\left[f^{+}+f^{-}\right](m)=C^{+}\left[f^{+}\right](m)+C^{-}\left[f^{-}\right](m):=C[f](m) .
$$

Therefore, the main problem lies in defining $C[\cdot]$ in the layers $(\underline{m},-1),(\underline{m}, 0),(\underline{m}, 1), \underline{m} \in \mathbb{Z}^{2}$. Here, for arbitrary $\underline{m} \in \mathbb{Z}^{2}$ we have

$$
\begin{aligned}
& 0=D_{h}^{+-} f^{+}(\underline{m},-1)=-\sum_{j=1}^{3}\left(e_{j}^{+} \partial^{+j} f^{+}(\underline{m},-1)+e_{j}^{-} \partial^{-j} f^{+}(\underline{m},-1)\right) \\
& =-e_{3}^{+} \partial^{+3} f^{+}(\underline{m},-1)-e_{3}^{-} \partial^{-3} f^{+}(\underline{m},-1)=e_{3}^{+}\left(f^{+}(\underline{m}, 0)-0\right)+e_{3}^{-}(0-0) \\
& =-e_{3}^{+} f^{+}(\underline{m}, 0),
\end{aligned}
$$

which implies $f^{+}(\underline{m}, 0)=e_{3}^{+}\left[A_{1}(\underline{m})+e_{3}^{-} B_{1}(\underline{m})\right]$, with $A_{1}, B_{1}$ being discrete functions taking values in the subalgebra $\mathbb{C}_{2}$ of $\mathbb{C}_{3}$, spanned by $\left\{e_{1}^{+}, e_{1}^{-}, e_{2}^{+}, e_{2}^{-}\right\}$.

Moreover, we get

$$
\begin{aligned}
& C^{-}\left[f^{+}\right](\underline{m}, 1)=\sum_{\underline{n} \in \mathbb{Z}^{2}}\left[E_{h}^{-+}(\underline{n}-\underline{m},-2) e_{3}^{+} f^{+}(\underline{n}, 0)+E_{h}^{-+}(\underline{n}-\underline{m},-1) e_{3}^{-} f^{+}(\underline{n},-1)\right] h^{3} \\
& =\sum_{\underline{n} \in \mathbb{Z}^{2}} E_{h}^{-+}(\underline{n}-\underline{m},-2) e_{3}^{+} f^{+}(\underline{n}, 0) h^{3} \\
& =\sum_{\underline{n} \in \mathbb{Z}^{2}} E_{h}^{-+}(\underline{n}-\underline{m},-2) e_{3}^{+} e_{3}^{+}\left[A_{1}(\underline{m})+e_{3}^{-} B_{1}(\underline{m})\right] h^{3}=0 .
\end{aligned}
$$

Likewise, for function $f^{-}$we obtain

$$
0=D_{h}^{+-} f^{-}(\underline{m}, 1)=-e_{3}^{+}(0-0)-e_{3}^{-}\left(f^{-}(\underline{m}, 0)-0\right)=-e_{3}^{-} f^{-}(\underline{m}, 0), \underline{m} \in \mathbb{Z}^{2},
$$

which implies $f^{-}(\underline{m}, 0)=e_{3}^{-}\left[A_{2}(\underline{m})+e_{3}^{+} B_{2}(\underline{m})\right]$, with $A_{2}, B_{2}$ being discrete functions taking values in the subalgebra $\mathbb{C}_{2}$ of $\mathbb{C}_{3}$, spanned by $\left\{e_{1}^{+}, e_{1}^{-}, e_{2}^{+}, e_{2}^{-}\right\}$. 
Hence $C^{+}\left[f^{-}\right](\underline{m}, 1)=0$ for arbitrary $\underline{m} \in \mathbb{Z}^{2}$.

Therefore, for arbitrary $\underline{m} \in \mathbb{Z}^{2}$, we obtain

$$
\begin{aligned}
& C[f](\underline{m}, 1)=\left(C^{+}+C^{-}\right)\left[f^{+}+f^{-}\right](\underline{m}, 1)=C^{+}\left[f^{+}\right](\underline{m}, 1)+C^{-}\left[f^{+}\right](\underline{m}, 1) \\
& +C^{+}\left[f^{-}\right](\underline{m}, 1)+C^{-}\left[f^{-}\right](\underline{m}, 1)=C^{+}\left[f^{+}\right](\underline{m}, 1)+C^{-}\left[f^{-}\right](\underline{m}, 1) .
\end{aligned}
$$

Finally, by similar reasoning, we get

$$
C^{-}\left[f^{+}\right](\underline{m}, 0)=C^{+}\left[f^{-}\right](\underline{m}, 0)=0=C^{-}\left[f^{+}\right](\underline{m},-1)=C^{+}\left[f^{-}\right](\underline{m},-1), \underline{m} \in \mathbb{Z}^{2} .
$$

So that (3.24) also holds for the layers $(\underline{m}, 0)$ and $(\underline{m},-1)$ with $\underline{m} \in \mathbb{Z}^{2}$.

Remark 3.7 This construction of the Cauchy transform can obviously be extended to an arbitrary decomposition of function $f$ into upper and lower monogenics that exempt regularity on a finite number of layers surrounding the 0-layer.

In conclusion, for arbitrary function $f \in \mathfrak{F}\left(\mathbb{Z}^{3}\right)$, we have the discrete Cauchy transform as follows

$$
C[f](m)= \begin{cases}C^{+}\left[f^{+}\right](m), & \text { if } m_{3} \geqslant 1, \\ C^{-}\left[f^{-}\right](m), & \text { if } m_{3} \leqslant-1 .\end{cases}
$$

Moreover, for the case of $m_{3}=0, \underline{m} \in \mathbb{Z}^{2}$, we get the discrete Cauchy transform as

$$
\begin{aligned}
0= & C[f](\underline{m}, 0)=C^{+}\left[f^{+}\right](\underline{m}, 0)+C^{-}\left[f^{-}\right](\underline{m}, 0) \\
= & -\sum_{\underline{n} \in \mathbb{Z}^{2}}\left[E_{h}^{-+}(\underline{n}-\underline{m}, 0) e_{3}^{+} f^{+}(\underline{n}, 1)+E_{h}^{-+}(\underline{n}-\underline{m}, 1) e_{3}^{-} f^{+}(\underline{n}, 0)\right. \\
& \left.+E_{h}^{-+}(\underline{n}-\underline{m},-1) e_{3}^{+} f^{-}(\underline{n}, 0)+E_{h}^{-+}(\underline{n}-\underline{m}, 0) e_{3}^{-} f^{-}(\underline{n},-1)\right] h^{3} .
\end{aligned}
$$

From Theorem 3.3 we obtain a discrete equivalent of the boundary behaviour of monogenic functions. From formula (3.13) we have for the boundary values (at the layer $m_{3}=1$ of a function which is discrete monogenic in the upper half plane

$$
-\sum_{\underline{n} \in \mathbb{Z}^{2}}\left[E_{h}^{-+}(\underline{n}-\underline{m},-1) e_{3}^{+} f(\underline{n}, 1)+E_{h}^{-+}(\underline{n}-\underline{m}, 0) e_{3}^{-} f(\underline{n}, 0)\right] h^{3}=f(\underline{m}, 1),
$$

while from formula (3.14) for the boundary values $\left(m_{3}=-1\right)$ of a function which is discrete monogenic in the lower half plane we get

$$
\sum_{\underline{n} \in \mathbb{Z}^{2}}\left[E_{h}^{-+}(\underline{n}-\underline{m}, 0) e_{3}^{+} f(\underline{n}, 0)+E_{h}^{-+}(\underline{n}-\underline{m}, 1) e_{3}^{-} f(\underline{n},-1)\right] h^{3}=f(\underline{m},-1),
$$

Additionally, we have for the values in the 0-layer in case of a function which is monogenic in the (complete) upper half space

$$
\sum_{\underline{n} \in \mathbb{Z}^{2}}\left[E_{h}^{-+}(\underline{n}-\underline{m}, 0) e_{3}^{+} f(\underline{n}, 1)+E_{h}^{-+}(\underline{n}-\underline{m}, 1) e_{3}^{-} f(\underline{n}, 0)\right] h^{3}=0
$$

and

$$
\sum_{\underline{n} \in \mathbb{Z}^{2}}\left[E_{h}^{-+}(\underline{n}-\underline{m},-1) e_{3}^{+} f(\underline{n}, 0)+E_{h}^{-+}(\underline{n}-\underline{m}, 0) e_{3}^{-} f(\underline{n},-1)\right] h^{3}=0 .
$$

for a function which is monogenic in the (complete) lower half space. 


\section{Reconstruction on the boundary layers}

From the last formulae in the previous section we can observe that the boundary condition involves function values not only in the 0-layer, but also in the layers above and below. Now, this raises the question how far conditions (3.27) and (3.27) can be solved in the two upper, respectively two lower layers of the boundary? This will give us also the discrete equivalent of the Plemelj projections and the Hardy spaces.

For studying the above equation we need the corresponding Fourier symbols.

Lemma 4.1 The Fourier symbols of the fundamental solution on the layers $-1,0,1$ are given by

$$
\begin{aligned}
\mathcal{F}_{h} E^{-+}(\underline{\xi}, 0)= & \frac{\underline{\widetilde{\xi}}_{-}}{\underline{d}} \frac{1}{\sqrt{4+h^{2} \underline{d}^{2}}}+\left(e_{3}^{+}-e_{3}^{-}\right)\left(\frac{1}{2}-\frac{h \underline{d}}{2 \sqrt{4+h^{2} \underline{d}^{2}}}\right) \\
\mathcal{F}_{h} E^{-+}(\underline{\xi}, 1)= & \frac{\underline{\widetilde{\xi}}_{-}}{\underline{d}}\left(\frac{2+\underline{d}^{2} h^{2}}{2 \sqrt{4+h^{2} \underline{d}^{2}}}-\frac{h \underline{d}}{2}\right)-e_{3}^{-}\left(-\frac{3 h \underline{d}+h^{3} \underline{d}^{3}}{2 \sqrt{4+h^{2} \underline{d}^{2}}}+\frac{h^{2} \underline{d}^{2}}{2}+\frac{1}{2}\right) \\
& +e_{3}^{+}\left(\frac{h \underline{d}}{2 \sqrt{4+h^{2} \underline{d}^{2}}}-\frac{1}{2}\right), \\
\mathcal{F}_{h} E^{-+}(\underline{\xi},-1)= & \frac{\widetilde{\xi}_{-}}{\underline{d}}\left(\frac{2+\underline{d}^{2} h^{2}}{2 \sqrt{4+h^{2} \underline{d}^{2}}}-\frac{h \underline{d}}{2}\right)+e_{3}^{+}\left(-\frac{3 h \underline{d}+h^{3} \underline{d}^{3}}{2 \sqrt{4+h^{2} \underline{d}^{2}}}+\frac{h^{2} \underline{d}^{2}}{2}+\frac{1}{2}\right) \\
& -e_{3}^{-}\left(\frac{h \underline{d}}{2 \sqrt{4+h^{2} \underline{d}^{2}}}-\frac{1}{2}\right) .
\end{aligned}
$$

Proof: From Section 2, we know that the Fourier symbol of the fundamental solution $E^{-+}(n)$ for arbitrary $n \in \mathbb{Z}^{3}$ is given by $\frac{\widetilde{\xi}_{-}}{d^{2}}$ with $\widetilde{\xi}_{-}=\sum_{j=1}^{3} e_{j}^{+} \xi_{-j}^{D}+e_{j}^{-} \xi_{+j}^{D}$. Therefore, the Fourier symbol of $E^{-+}\left(\underline{n}, n_{3}\right)$ for arbitrary $n \in \mathbb{Z}^{2}, n_{3}$ fixed is given by

$$
\begin{gathered}
\mathcal{F}_{h} E^{-+}\left(\underline{\eta}, x_{3}\right)=\sum_{\underline{x} \in \mathbb{Z}^{2}} e^{i<\underline{x}, \underline{\eta}>} \frac{1}{(2 \pi)^{3}} \int_{[-\pi / h, \pi / h]^{3}} e^{-i<x, \xi>\frac{\widetilde{\xi}_{-}}{d^{2}} d \xi} \\
=\sum_{\underline{x} \in \mathbb{Z}^{2}} e^{i<\underline{x}, \underline{\eta}>} \frac{1}{(2 \pi)^{3}} \int_{[-\pi / h, \pi / h]^{3}} e^{-i<x, \xi>} \frac{\underline{\widetilde{\xi}}_{-}+\underline{\widetilde{\xi}}_{3}}{\underline{d}^{2}+\frac{4}{h^{2}} \sin ^{2} \frac{\xi_{3} h}{2}} d \xi \\
=\frac{1}{(2 \pi)^{2}} \int_{[-\pi / h, \pi / h]^{2}} \sum_{\underline{x} \in \mathbb{Z}^{2}} e^{i<\underline{x}, \underline{\eta}-\underline{\xi}>} \frac{1}{2 \pi} \int_{-\frac{\pi}{h}}^{\frac{\pi}{h}}\left(\frac{e^{-i x_{3} \xi_{3}} \underline{\widetilde{\xi}}_{-}}{\underline{d}^{2}+\frac{4}{h^{2}} \sin ^{2} \frac{\xi_{3} h}{2}}+\frac{e^{-i x_{3} \xi_{3}} \underline{\tilde{\xi}}_{3}}{\underline{d}^{2}+\frac{4}{h^{2}} \sin ^{2} \frac{\xi_{3} h}{2}}\right) d \xi_{3} d \underline{\xi},
\end{gathered}
$$

where $\underline{d}^{2}=\frac{4}{h^{2}} \sum_{j=1}^{2} \sin ^{2} \frac{\xi_{j} h}{2}$ and $\underline{\tilde{\xi}}_{-}=\sum_{j=1}^{2} e_{j}^{+} \xi_{-j}^{D}+e_{j}^{-} \xi_{+j}^{D}, \underline{\xi}_{3}=e_{3}^{+} \xi_{-3}^{D}+e_{3}^{-} \xi_{+3}^{D}$. We are interested in the Fourier symbol for $x_{3}=-1,0,1$. To this end we need the following integrals

$$
\int_{-\frac{\pi}{h}}^{\frac{\pi}{h}} \frac{1}{\underline{d}^{2}+\frac{4}{h^{2}} \sin ^{2} \frac{\xi_{3} h}{2}} d \xi_{3}=\left.\frac{2}{\underline{d}^{2}} \sqrt{\frac{\underline{d}^{2}}{4+h^{2} \underline{d}^{2}}} \arctan \frac{\tan \frac{h \xi_{3}}{2}}{\sqrt{\frac{h^{2} d^{2}}{4+h^{2} \underline{d}^{2}}}}\right|_{-\frac{\pi}{h}} ^{\frac{\pi}{h}}=\frac{2 \pi}{\underline{d} \sqrt{4+h^{2} \underline{d}^{2}}},
$$




$$
\begin{aligned}
& \int_{-\frac{\pi}{h}}^{\frac{\pi}{h}} \frac{2 h^{-1} \sin ^{2} \frac{h \xi_{3}}{2}}{\underline{d}^{2}+\frac{4}{h^{2}} \sin ^{2} \frac{\xi_{3} h}{2}} d \xi_{3}=\frac{h}{2} \int_{-\frac{\pi}{h}}^{\frac{\pi}{h}}\left(1-\frac{1}{1+\frac{4}{h^{2} \underline{d}^{2}} \sin ^{2} \frac{\xi_{3} h}{2}}\right) d \xi_{3}=\pi-\frac{h \underline{d} \pi}{\sqrt{4+h^{2} \underline{d}^{2}}}, \\
& \int_{-\frac{\pi}{h}}^{\frac{\pi}{h}} \frac{h^{-1} \cosh \xi_{3}}{\underline{d}^{2}+\frac{4}{h^{2}} \sin ^{2} \frac{\xi_{3} h}{2}} d \xi_{3}=\int_{-\frac{\pi}{h}}^{\frac{\pi}{h}} \frac{h^{-1}\left(1-2 \sin ^{2} \frac{h \xi_{3}}{2}\right)}{\underline{d}^{2}+\frac{4}{h^{2}} \sin ^{2} \frac{\xi_{3} h}{2}} d \xi_{3}=\int_{-\frac{\pi}{h}}^{\frac{\pi}{h}} \frac{h^{-1}}{\underline{d}^{2}+\frac{4}{h^{2}} \sin ^{2} \frac{\xi_{3} h}{2}} d \xi_{3} \\
& -\int_{-\frac{\pi}{h}}^{\frac{\pi}{h}} \frac{2 h^{-1} \sin ^{2} \frac{h \xi_{3}}{2}}{\underline{d}^{2}+\frac{4}{h^{2}} \sin ^{2} \frac{\xi_{3} h}{2}} d \xi_{3}=\frac{2 \pi}{h \underline{d} \sqrt{4+h^{2} \underline{d}^{2}}}-\pi+\frac{h \underline{d} \pi}{\sqrt{4+h^{2} \underline{d}^{2}}},
\end{aligned}
$$

and

$$
\begin{aligned}
& \int_{-\frac{\pi}{h}}^{\frac{\pi}{h}} \frac{h^{-1} \cos 2 h \xi_{3}}{d^{2}+\frac{4}{h^{2}} \sin ^{2} \frac{\xi_{3} h}{2}} d \xi_{3}=\frac{1}{d^{2} h} \int_{-\frac{\pi}{2}}^{\frac{\pi}{2}} \frac{\cos 4 t}{1+\frac{4}{d^{2} h^{2}} \sin ^{2} t} \frac{2}{h} d t \\
& =\frac{2}{d^{2} h^{2}} \int_{-\frac{\pi}{2}}^{\frac{\pi}{2}} \frac{1-8 \cos ^{2} t \sin ^{2} t}{1+\frac{4}{d^{2} h^{2}} \sin ^{2} t} d t=2 \int_{-\frac{\pi}{2}}^{\frac{\pi}{2}} \frac{\frac{1}{d^{2} h^{2}}+2-2-\frac{8}{d^{2} h^{2}} \cos ^{2} t \sin ^{2} t}{1+\frac{4}{d^{2} h^{2}} \sin ^{2} t} d t \\
& =2\left[\int_{-\frac{\pi}{2}}^{\frac{\pi}{2}} \frac{\frac{1}{d^{2} h^{2}}+2}{1+\frac{4}{d^{2} h^{2}} \sin ^{2} t} d t-2 \int_{-\frac{\pi}{2}}^{\frac{\pi}{2}} \frac{\sin ^{2} t+\cos ^{2} t+\frac{4}{d^{2} h^{2}} \cos ^{2} t \sin ^{2} t}{1+\frac{4}{d^{2} h^{2}} \sin ^{2} t} d t\right] \\
& =2\left[\left(\frac{1}{d^{2} h^{2}}+2\right) \int_{-\frac{\pi}{2}}^{\frac{\pi}{2}} \frac{1}{1+\frac{4}{d^{2} h^{2}} \sin ^{2} t} d t-2 \int_{-\frac{\pi}{2}}^{\frac{\pi}{2}} \frac{\sin ^{2}(t)}{1+\frac{4}{d^{2} h^{2}} \sin ^{2} t} d t-2 \int_{-\frac{\pi}{2}}^{\frac{\pi}{2}} \cos ^{2} t d t\right] \\
& =2\left[\left(\frac{1}{d^{2} h^{2}}+2\right) \frac{\pi h \underline{d}}{\sqrt{4+h^{2} \underline{d}^{2}}}+\frac{h^{3} \underline{d^{3}}}{2} \frac{\pi}{\sqrt{4+h^{2} \underline{d}^{2}}}-\frac{h^{2} \underline{d}^{2}}{2} \pi-\pi\right] \\
& =2\left[\left(\frac{1}{\underline{d}^{2}}+2 \underline{d} h+\frac{h^{3} \underline{d^{3}}}{2}\right) \frac{\pi}{\sqrt{4+h^{2} \underline{d}^{2}}}-\frac{h^{2} \underline{d}^{2}}{2} \pi-\pi\right] .
\end{aligned}
$$

Now, for $x_{3}=0$ we get

$$
\begin{aligned}
& \mathcal{F}_{h} E^{-+}(\underline{\eta}, 0)=\frac{1}{(2 \pi)^{2}} \int_{[-\pi / h, \pi / h]^{2}} \sum_{\underline{x} \in \mathbb{Z}^{2}} e^{i<\underline{x}, \underline{\eta}-\underline{\xi}>} \frac{1}{2 \pi} \int_{-\frac{\pi}{h}}^{\frac{\pi}{h}}\left(\frac{\underline{\widetilde{\xi}}_{-}}{\underline{d}^{2}+\frac{4}{h^{2}} \sin ^{2} \frac{\xi_{3} h}{2}}+\frac{\underline{\widetilde{\xi}}_{3}}{\underline{d}^{2}+\frac{4}{h^{2}} \sin ^{2} \frac{\xi_{3} h}{2}}\right) d \xi_{3} d \underline{\xi} \\
& =\frac{1}{(2 \pi)^{2}} \int_{[-\pi / h, \pi / h]^{2}} \sum_{\underline{x} \in \mathbb{Z}^{2}} e^{i<\underline{x}, \underline{\eta}-\underline{\xi}>} \frac{1}{2 \pi} \int_{-\frac{\pi}{h}}^{\frac{\pi}{h}}\left(\frac{\underline{\xi}_{-}}{\underline{d}^{2}+\frac{4}{h^{2}} \sin ^{2} \frac{\xi_{3} h}{2}}+\frac{e_{3}^{+}\left(1-e^{i h \xi_{3}}\right)-e_{3}^{-}\left(1-e^{-i h \xi_{3}}\right)}{h\left(\underline{d}^{2}+\frac{4}{h^{2}} \sin ^{2} \frac{\xi_{3} h}{2}\right)}\right) d \xi_{3} d \underline{\xi} \\
& =\frac{1}{(2 \pi)^{2}} \int_{[-\pi / h, \pi / h]^{2}} \sum_{\underline{x} \in \mathbb{Z}^{2}} e^{i<\underline{x}, \underline{\eta}-\underline{\xi}>} \frac{1}{2 \pi} \int_{-\frac{\pi}{h}}^{\frac{\pi}{h}}\left(\frac{\underline{\tilde{\xi}}_{-}}{\underline{d}^{2}+\frac{4}{h^{2}} \sin ^{2} \frac{\xi_{3} h}{2}}+\left(e_{3}^{+}-e_{3}^{-}\right) \frac{1-\cos h \xi_{3}}{h\left(\underline{d}^{2}+\frac{4}{h^{2}} \sin ^{2} \frac{\xi_{3} h}{2}\right)}\right) d \xi_{3} d \underline{\xi} \\
& =\frac{1}{(2 \pi)^{2}} \int_{[-\pi / h, \pi / h]^{2}} \sum_{\underline{x} \in \mathbb{Z}^{2}} e^{i<\underline{x}, \underline{\eta}-\underline{\xi}>} \frac{1}{2 \pi} \int_{-\frac{\pi}{h}}^{\frac{\pi}{h}}\left(\frac{\underline{\widetilde{\xi}}_{-}}{\underline{d}^{2}+\frac{4}{h^{2}} \sin ^{2} \frac{\xi_{3} h}{2}}+\left(e_{3}^{+}-e_{3}^{-}\right) \frac{2 h^{-1} \sin ^{2} \frac{h \xi_{3}}{2}}{\underline{d}^{2}+\frac{4}{h^{2}} \sin ^{2} \frac{\xi_{3} h}{2}}\right) d \xi_{3} d \underline{\xi} .
\end{aligned}
$$

This results in

$$
\mathcal{F}_{h} E^{-+}(\underline{\xi}, 0)=\frac{\widetilde{\xi}_{-}}{\underline{d}} \frac{1}{\sqrt{4+h^{2} \underline{d}^{2}}}+\left(e_{3}^{+}-e_{3}^{-}\right)\left(\frac{1}{2}-\frac{h \underline{d}}{2 \sqrt{4+h^{2} \underline{d}^{2}}}\right) .
$$


Now, for the case $x_{3}=1$ we have

$$
\begin{aligned}
& \mathcal{F}_{h} E^{-+}(\underline{\eta}, 1)=\frac{1}{(2 \pi)^{2}} \int_{[-\pi / h, \pi / h]^{2}} \sum_{\underline{x} \in \mathbb{Z}^{2}} e^{i<\underline{x}, \underline{\eta}-\underline{\xi}>} \frac{1}{2 \pi} \int_{-\frac{\pi}{h}}^{\frac{\pi}{h}} e^{-i \xi_{3} h}\left(\frac{\underline{\tilde{\xi}}_{-}}{\underline{d}^{2}+\frac{4}{h^{2}} \sin ^{2} \frac{\xi_{3} h}{2}}+\frac{\widetilde{\xi}_{3}}{\underline{d}^{2}+\frac{4}{h^{2}} \sin ^{2} \frac{\xi_{3} h}{2}}\right) d \xi_{3} d \underline{\xi} \\
& =\frac{1}{(2 \pi)^{2}} \int_{[-\pi / h, \pi / h]^{2}} \sum_{\underline{x} \in \mathbb{Z}^{2}} e^{i<\underline{x}, \underline{\eta}-\underline{\xi}>} \frac{1}{2 \pi} \int_{-\frac{\pi}{h}}^{\frac{\pi}{h}} e^{-i \xi_{3} h}\left(\frac{\underline{\tilde{\xi}}_{-}}{\underline{d}^{2}+\frac{4}{h^{2}} \sin ^{2} \frac{\xi_{3} h}{2}}+\frac{e_{3}^{+}\left(1-e^{i h \xi_{3}}\right)-e_{3}^{-}\left(1-e^{-i h \xi_{3}}\right)}{h\left(\underline{d}^{2}+\frac{4}{h^{2}} \sin ^{2} \frac{\xi_{3} h}{2}\right)}\right) d \xi_{3} d \underline{\xi} \\
& =\frac{1}{(2 \pi)^{2}} \int_{[-\pi / h, \pi / h]^{2}} \sum_{\underline{x} \in \mathbb{Z}^{2}} e^{i<\underline{x}, \underline{\eta}-\underline{\xi}>} \frac{1}{2 \pi} \int_{-\frac{\pi}{h}}^{\frac{\pi}{h}} \frac{e^{-i \xi_{3} h} \underline{\widetilde{\xi}}_{-}}{\underline{d}^{2}+\frac{4}{h^{2}} \sin ^{2} \frac{\xi_{3} h}{2}}+\frac{e_{3}^{+}\left(1-e^{i h \xi_{3}}\right)-e_{3}^{-}\left(1-e^{-i h \xi_{3}}\right)}{h\left(\underline{d}^{2}+\frac{4}{h^{2}} \sin ^{2} \frac{\xi_{3} h}{2}\right)} e^{-i \xi_{3} h} d \xi_{3} d \underline{\xi} \\
& =\frac{1}{(2 \pi)^{2}} \int_{[-\pi / h, \pi / h]^{2}} \sum_{\underline{x} \in \mathbb{Z}^{2}} e^{i<\underline{x}, \underline{\eta}-\underline{\xi}>} \frac{1}{2 \pi} \int_{-\frac{\pi}{h}}^{\frac{\pi}{h}}\left(\frac{\widetilde{\xi}_{-} \cos \xi_{3} h}{\underline{d}^{2}+\frac{4}{h^{2}} \sin ^{2} \frac{\xi_{3} h}{2}}+\frac{e_{3}^{+}\left(e^{-i \xi_{3} h}-1\right)-e_{3}^{-}\left(e^{-i \xi_{3} h}-e^{-2 i \xi_{3} h}\right)}{h\left(\underline{d}^{2}+\frac{4}{h^{2}} \sin ^{2} \frac{\xi_{3} h}{2}\right)}\right) d \xi_{3} d \underline{\xi} \\
& =\frac{1}{(2 \pi)^{2}} \int_{[-\pi / h, \pi / h]^{2}} \sum_{\underline{x} \in \mathbb{Z}^{2}} e^{i<\underline{x}, \underline{\eta}-\underline{\xi}>} \frac{1}{2 \pi} \int_{-\frac{\pi}{h}}^{\frac{\pi}{h}}\left(\frac{\underline{\tilde{\xi}}_{-} \cos \xi_{3} h}{\underline{d}^{2}+\frac{4}{h^{2}} \sin ^{2} \frac{\xi_{3} h}{2}}+e_{3}^{+}\left(\frac{h^{-1} \cos h \xi_{3}}{\underline{d}^{2}+\frac{4}{h^{2}} \sin ^{2} \frac{\xi_{3} h}{2}}-\frac{h^{-1}}{\underline{d}^{2}+\frac{4}{h^{2}} \sin ^{2} \frac{\xi_{3} h}{2}}\right)\right. \\
& \left.-e_{3}^{-}\left(\frac{h^{-1} \cos h \xi_{3}}{\underline{d}^{2}+\frac{4}{h^{2}} \sin ^{2} \frac{\xi_{3} h}{2}}-\frac{h^{-1} \cos 2 h \xi_{3}}{\underline{d}^{2}+\frac{4}{h^{2}} \sin ^{2} \frac{\xi_{3} h}{2}}\right)\right) d \xi_{3} d \underline{\xi} .
\end{aligned}
$$

Here we get

$$
\begin{aligned}
& \mathcal{F}_{h} E^{-+}(\underline{\xi}, 1)=\frac{1}{2 \pi}\left[\underline{\widetilde{\xi}}_{-}\left(\frac{2 \pi}{\underline{d} \sqrt{4+h^{2} \underline{d}^{2}}}-\pi h+\frac{h^{2} \underline{d} \pi}{\sqrt{4+h^{2} \underline{d}^{2}}}\right)-e_{3}^{-}\left(\frac{2 \pi}{h \underline{d} \sqrt{4+h^{2} \underline{d}^{2}}}-\pi+\frac{h \underline{d} \pi}{\sqrt{4+h^{2} \underline{d}^{2}}}\right.\right. \\
& \left.-2\left[\left(\frac{1}{\underline{d} h}+2 \underline{d} h+\frac{h^{3} \underline{d}^{3}}{2}\right) \frac{\pi}{\sqrt{4+h^{2} \underline{d}^{2}}}-\frac{h^{2} \underline{d}^{2}}{2} \pi-\pi\right]\right)+e_{3}^{+}\left(\frac{2 \pi}{h \underline{d} \sqrt{4+h^{2} \underline{d}^{2}}}-\pi+\frac{h \underline{d} \pi}{\sqrt{4+h^{2} \underline{d}^{2}}}-\frac{2 \pi}{h \underline{d} \sqrt{4+h^{2} \underline{d}^{2}}}\right) \\
& =\frac{\widetilde{\xi}_{-}}{\underline{d}}\left(\frac{2+\underline{d}^{2} h^{2}}{2 \sqrt{4+h^{2} \underline{d}^{2}}}-\frac{h \underline{d}}{2}\right)-e_{3}^{-}\left(-\frac{3 h \underline{d}+h^{3} \underline{d}^{3}}{2 \sqrt{4+h^{2} \underline{d}^{2}}}+\frac{h^{2} \underline{d}^{2}}{2}+\frac{1}{2}\right)+e_{3}^{+}\left(\frac{h \underline{d}}{2 \sqrt{4+h^{2} \underline{d}^{2}}}-\frac{1}{2}\right) .
\end{aligned}
$$

Finally, for the case $x_{3}=-1$ we obtain

$$
\begin{aligned}
& \mathcal{F}_{h} E^{-+}(\underline{\xi},-1)=\frac{1}{(2 \pi)^{2}} \int_{[-\pi / h, \pi / h]^{2}} \sum_{\underline{x} \in \mathbb{Z}^{2}} e^{i<\underline{x}, \underline{\eta}-\underline{\xi}>} \frac{1}{2 \pi} \int_{-\frac{\pi}{h}}^{\frac{\pi}{h}} e^{i \xi_{3} h}\left(\frac{\underline{\xi}_{-}}{\underline{d}^{2}+\frac{4}{h^{2}} \sin ^{2} \frac{\xi_{3} h}{2}}+\frac{\underline{\underline{\xi}}_{3}}{\underline{d}^{2}+\frac{4}{h^{2}} \sin ^{2} \frac{\xi_{3} h}{2}}\right) d \xi_{3} d \underline{\xi} \\
& =\frac{1}{(2 \pi)^{2}} \int_{[-\pi / h, \pi / h]^{2}} \sum_{\underline{x} \in \mathbb{Z}^{2}} e^{i<\underline{x}, \underline{\eta}-\underline{\xi}>} \frac{1}{2 \pi} \int_{-\frac{\pi}{h}}^{\frac{\pi}{h}} e^{i \xi_{3} h}\left(\frac{\underline{\widetilde{\xi}}_{-}}{\underline{d}^{2}+\frac{4}{h^{2}} \sin ^{2} \frac{\xi_{3} h}{2}}+\frac{e_{3}^{+}\left(1-e^{i h \xi_{3}}\right)-e_{3}^{-}\left(1-e^{-i h \xi_{3}}\right)}{h\left(\underline{d}^{2}+\frac{4}{h^{2}} \sin ^{2} \frac{\xi_{3} h}{2}\right)}\right) d \xi_{3} d \underline{\xi} \\
& =\frac{1}{(2 \pi)^{2}} \int_{[-\pi / h, \pi / h]^{2}} \sum_{\underline{x} \in \mathbb{Z}^{2}} e^{i<\underline{x}, \underline{\eta}-\underline{\xi}>} \frac{1}{2 \pi} \int_{-\frac{\pi}{h}}^{\frac{\pi}{h}} \frac{e^{i \xi_{3} h} \underline{\widetilde{\xi}}_{-}}{\underline{d}^{2}+\frac{4}{h^{2}} \sin ^{2} \frac{\xi_{3} h}{2}}+\frac{e_{3}^{+}\left(e^{i h \xi_{3}}-e^{2 i h \xi_{3}}\right)-e_{3}^{-}\left(e^{i h \xi_{3}}-1\right)}{h\left(\underline{d}^{2}+\frac{4}{h^{2}} \sin ^{2} \frac{\xi_{3} h}{2}\right)} d \xi_{3} d \underline{\xi} \\
& =\frac{1}{(2 \pi)^{2}} \int_{[-\pi / h, \pi / h]^{2}} \sum_{\underline{x} \in \mathbb{Z}^{2}} e^{i<\underline{x}, \underline{\eta}-\underline{\xi}>} \frac{1}{2 \pi} \int_{-\frac{\pi}{h}}^{\frac{\pi}{h}}\left(\frac{\underline{\xi}_{-} \cos \xi_{3} h}{\underline{d}^{2}+\frac{4}{h^{2}} \sin ^{2} \frac{\xi_{3} h}{2}}+e_{3}^{+}\left(\frac{h^{-1} \cos h \xi_{3}}{\underline{d}^{2}+\frac{4}{h^{2}} \sin ^{2} \frac{\xi_{3} h}{2}}-\frac{h^{-1} \cos 2 h \xi_{3}}{\underline{d}^{2}+\frac{4}{h^{2}} \sin ^{2} \frac{\xi_{3} h}{2}}\right)\right. \\
& \left.-e_{3}^{-}\left(\frac{h^{-1} \cos h \xi_{3}}{\underline{d}^{2}+\frac{4}{h^{2}} \sin ^{2} \frac{\xi_{3} h}{2}}-\frac{h^{-1}}{\underline{d}^{2}+\frac{4}{h^{2}} \sin ^{2} \frac{\xi_{3} h}{2}}\right)\right) d \xi_{3} d \underline{\xi} .
\end{aligned}
$$


Hence, we get

$$
\begin{aligned}
& \mathcal{F}_{h} E^{-+}(\underline{\xi},-1)=\frac{1}{2 \pi}\left[\underline{\widetilde{\xi}}_{-}\left(\frac{2 \pi}{\underline{d} \sqrt{4+h^{2} \underline{d}^{2}}}-h \pi+\frac{h^{2} \underline{d} \pi}{\sqrt{4+h^{2} \underline{d}^{2}}}\right)\right. \\
& +e_{3}^{+}\left(\frac{2 \pi}{h \underline{d} \sqrt{4+h^{2} \underline{d}^{2}}}-\pi+\frac{h \underline{d} \pi}{\sqrt{4+h^{2} \underline{d}^{2}}}\right. \\
& \left.-2\left(\left(\frac{1}{\underline{d h}}+2 \underline{d} h+\frac{h^{3} \underline{d}^{3}}{2}\right) \frac{\pi}{\sqrt{4+h^{2} \underline{d}^{2}}}-\frac{h^{2} \underline{d}^{2}}{2} \pi-\pi\right)\right) \\
& \left.-e_{3}^{-}\left(\frac{2 \pi}{h \underline{d} \sqrt{4+h^{2} \underline{d}^{2}}}-\pi+\frac{h \underline{d} \pi}{\sqrt{4+h^{2} \underline{d}^{2}}}-\frac{2 \pi}{h \underline{d} \sqrt{4+h^{2} \underline{d}^{2}}}\right)\right] \\
& =\frac{\underline{\widetilde{\xi}}_{-}}{\underline{d}^{2}}\left(\frac{2+\underline{d}^{2} h^{2}}{2 \sqrt{4+h^{2} \underline{d}^{2}}}-\frac{h \underline{d}}{2}\right)+e_{3}^{+}\left(-\frac{3 h \underline{d}+h^{3} \underline{d}^{3}}{2 \sqrt{4+h^{2} \underline{d}^{2}}}+\frac{h^{2} \underline{d}^{2}}{2}+\frac{1}{2}\right)-e_{3}^{-}\left(\frac{h \underline{d}}{2 \sqrt{4+h^{2} \underline{d}^{2}}}-\frac{1}{2}\right) .
\end{aligned}
$$

Now, conditions (3.27) and (3.27) can be written on the Fourier side as

$$
\mathcal{F}_{h} E^{-+}(\underline{\xi},-1) e_{3}^{+} \mathcal{F}_{h} f^{+}(\underline{\xi}, 1)+\mathcal{F}_{h} E^{-+}(\underline{\xi}, 0) e_{3}^{-} \mathcal{F}_{h} f^{+}(\underline{\xi}, 0)=-\mathcal{F}_{h} f^{+}(\underline{\xi}, 1) \text {. }
$$

as well as

$$
\mathcal{F}_{h} E^{-+}(\underline{\xi}, 1) e_{3}^{-} \mathcal{F}_{h} f^{-}(\underline{\xi},-1)+\mathcal{F}_{h} E^{-+}(\underline{\xi}, 0) e_{3}^{+} \mathcal{F}_{h} f^{-}(\underline{\xi}, 0)=\mathcal{F}_{h} f^{-}(\underline{\xi},-1) .
$$

while for the zero layer we have

$$
\begin{aligned}
& \mathcal{F}_{h} E^{-+}(\underline{\xi}, 1) e_{3}^{-} \mathcal{F}_{h} f^{+}(\underline{\xi}, 0)+\mathcal{F}_{h} E^{-+}(\underline{\xi}, 0) e_{3}^{+} \mathcal{F}_{h} f^{+}(\underline{\xi}, 1)=0, \\
& \mathcal{F}_{h} E^{-+}(\underline{\xi},-1) e_{3}^{+} \mathcal{F}_{h} f^{-}(\underline{\xi}, 0)+\mathcal{F}_{h} E^{-+}(\underline{\xi}, 0) e_{3}^{-} \mathcal{F}_{h} f^{-}(\underline{\xi},-1)=0,
\end{aligned}
$$

respectively.

Therefore, using the above lemma the terms (4.32) and (4.33) can be written in Fourier domain as

$$
\begin{aligned}
& \left(\frac{\underline{\widetilde{\xi}}_{-}}{\underline{d}}\left(\frac{2+\underline{d}^{2} h^{2}}{2 \sqrt{4+h^{2} \underline{d}^{2}}}-\frac{h \underline{d}}{2}\right)-e_{3}^{-}\left(\frac{h \underline{d}}{2 \sqrt{4+h^{2} \underline{d}^{2}}}-\frac{1}{2}\right)\right) e_{3}^{+} \mathcal{F}_{h} f^{+}(\underline{\xi}, 1) \\
& =-\mathcal{F}_{h} f^{+}(\underline{\xi}, 1)-\left(\frac{\underline{\xi}}{\underline{\underline{d}}} \frac{1}{\sqrt{4+h^{2} \underline{d}^{2}}}+e_{3}^{+}\left(\frac{1}{2}-\frac{h \underline{d}}{2 \sqrt{4+h^{2} \underline{d}^{2}}}\right)\right) e_{3}^{-} \mathcal{F}_{h} f^{+}(\underline{\xi}, 0),
\end{aligned}
$$

as well as

$$
\begin{aligned}
& \left(\frac{\widetilde{\widetilde{\xi}}_{-}}{\underline{d}}\left(\frac{2+\underline{d}^{2} h^{2}}{2 \sqrt{4+h^{2} \underline{d}^{2}}}-\frac{h \underline{d}}{2}\right)+e_{3}^{+}\left(\frac{h \underline{d}}{2 \sqrt{4+h^{2} \underline{d}^{2}}}-\frac{1}{2}\right)\right) e_{3}^{-} \mathcal{F}_{h} f^{-}(\underline{\xi},-1) \\
& =\mathcal{F}_{h} f^{-}(\underline{\xi},-1)-\left(\frac{\underline{\xi}}{\underline{d}} \frac{1}{\sqrt{4+h^{2} \underline{d}^{2}}}-e_{3}^{-}\left(\frac{1}{2}-\frac{h \underline{d}}{2 \sqrt{4+h^{2} \underline{d}^{2}}}\right)\right) e_{3}^{+} \mathcal{F}_{h} f^{-}(\underline{\xi}, 0) .
\end{aligned}
$$


while for the zero layer we get

$$
\begin{aligned}
& \left(\frac{\underline{\widetilde{\xi}}_{-}}{\underline{d}}\left(\frac{2+\underline{d}^{2} h^{2}}{2 \sqrt{4+h^{2} \underline{d}^{2}}}-\frac{h \underline{d}}{2}\right)+e_{3}^{+}\left(\frac{h \underline{d}}{2 \sqrt{4+h^{2} \underline{d}^{2}}}-\frac{1}{2}\right)\right) e_{3}^{-} \mathcal{F}_{h} f^{+}(\underline{\xi}, 0) \\
& =-\left(\frac{\underline{\widetilde{\xi}}_{-}}{\underline{d}} \frac{1}{\sqrt{4+h^{2} \underline{d}^{2}}}-e_{3}^{-}\left(\frac{1}{2}-\frac{h \underline{d}}{2 \sqrt{4+h^{2} \underline{d}^{2}}}\right)\right) e_{3}^{+} \mathcal{F}_{h} f^{+}(\underline{\xi}, 1),
\end{aligned}
$$

and

$$
\begin{aligned}
& \left(\frac{\widetilde{\xi}_{-}}{\underline{d}}\left(\frac{2+\underline{d}^{2} h^{2}}{2 \sqrt{4+h^{2} \underline{d}^{2}}}-\frac{h \underline{d}}{2}\right)-e_{3}^{-}\left(\frac{h \underline{d}}{2 \sqrt{4+h^{2} \underline{d}^{2}}}-\frac{1}{2}\right)\right) e_{3}^{+} \mathcal{F}_{h} f^{-}(\underline{\xi}, 0) \\
& =-\left(\frac{\underline{\xi}_{-}}{\underline{d}} \frac{1}{\sqrt{4+h^{2} \underline{d}^{2}}}+e_{3}^{+}\left(\frac{1}{2}-\frac{h \underline{d}}{2 \sqrt{4+h^{2} \underline{d}^{2}}}\right)\right) e_{3}^{-} \mathcal{F}_{h} f^{-}(\underline{\xi},-1),
\end{aligned}
$$

Now, to get conditions for our boundary values we have to study the solvability of equations (4.35) and (4.36). For this we introduce the following abreviations:

$$
A=\frac{\tilde{\widetilde{\xi}}_{-}}{\underline{d}}\left(\frac{\underline{d}^{2} h^{2}}{2 \sqrt{4+h^{2} \underline{d}^{2}}}-\frac{h \underline{d}}{2}\right), B=\frac{\tilde{\widetilde{\xi}}_{-}}{\underline{d}} \frac{1}{\sqrt{4+h^{2} \underline{d}^{2}}}, C=\frac{h \underline{d}}{2 \sqrt{4+h^{2} \underline{d}^{2}}}-\frac{1}{2} .
$$

We would like to point our that $C$ is a scalar operator while $A$ and $B$ are vector-valued operators. Since $e_{3}^{ \pm}$act like projectors $\left(\left(e_{3}^{ \pm}\right)^{2}=0\right.$ !) we can decompose our functions into

$$
\begin{gathered}
F^{ \pm}(\underline{\xi}, 0):=\mathcal{F}_{h} f^{ \pm}(\underline{\xi}, 0)=F_{1}^{ \pm, 0}+e_{3}^{+} F_{2}^{ \pm, 0}+e_{3}^{-} F_{3}^{ \pm, 0}+e_{3}^{+} e_{3}^{-} F_{4}^{ \pm, 0}, \\
F^{+}(\underline{\xi}, 1):=\mathcal{F}_{h} f^{+}(\underline{\xi}, 1)=F_{1}^{+, 1}+e_{3}^{+} F_{2}^{+, 1}+e_{3}^{-} F_{3}^{+, 1}+e_{3}^{+} e_{3}^{-} F_{4}^{+, 1}, \\
F^{-}(\underline{\xi},-1):=\mathcal{F}_{h} f^{-}(\underline{\xi},-1)=F_{1}^{-1}+e_{3}^{+} F_{2}^{-,-1}+e_{3}^{-} F_{3}^{-,-1}+e_{3}^{+} e_{3}^{-} F_{4}^{-,-1}
\end{gathered}
$$

where $F_{j}^{+, 0}, F_{j}^{-, 0}, F_{j}^{+, 1}, F_{j}^{-1}(j=1,2,3,4)$ belong to the subalgebra of $\mathbb{C}_{3}$ spanned by $\left\{e_{1}^{+}, e_{1}^{-}, e_{2}^{+}, e_{2}^{-}\right\}$.

Using this decomposition we can write the condition on layer $m_{3}=1$ as

$$
\begin{aligned}
& \left(\left(A+B-e_{3}^{-} C\right) e_{3}^{+}+1\right)\left(F_{1}^{+, 1}+e_{3}^{+} F_{2}^{+, 1}+e_{3}^{-} F_{3}^{+, 1}+e_{3}^{+} e_{3}^{-} F_{4}^{+, 1}\right) \\
& =-\left(B-e_{3}^{+} C\right) e_{3}^{-}\left(F_{1}^{+, 0}+e_{3}^{+} F_{2}^{+, 0}+e_{3}^{-} F_{3}^{+, 0}+e_{3}^{+} e_{3}^{-} F_{4}^{+, 0}\right),
\end{aligned}
$$

or in terms of our components

$$
\left\{\begin{array}{ccc}
F_{2}^{+, 1}-(A+B) F_{1}^{+, 1} & = & -C F_{2}^{+, 0}, \\
(C+1) F_{1}^{+, 1} & = & B F_{2}^{+, 0} \\
(C+1) F_{3}^{+, 1} & = & B\left(F_{1}^{+, 0}-F_{4}^{+, 0}\right) \\
C F_{1}^{+, 1}+(A+B) F_{3}^{+, 1}+F_{4}^{+, 1} & = & C\left(F_{1}^{+, 0}-F_{4}^{+, 0}\right)+B F_{2}^{+, 0} .
\end{array}\right.
$$

First of all, given the values of our function on layer $m_{3}=0$ we can obtain the values on layer $m_{3}=1$ by

$$
\left\{\begin{array}{ccc}
F_{1}^{+, 1}= & (C+1)^{-1} B F_{2}^{+, 0}, \\
F_{2}^{+, 1}= & -(C+1)^{-1}(C(C+1)-(A+B) B) F_{2}^{+, 0}, \\
F_{3}^{+, 1}= & (1+C)^{-1} B\left(F_{1}^{+, 0}-F_{4}^{+, 0}\right), \\
F_{4}^{+, 1}= & (1+C)^{-1}\left(B F_{2}^{+, 0}+(C(C+1)-(A+B) B)\left(F_{1}^{+, 0}-F_{4}^{+, 0}\right)\right) .
\end{array}\right.
$$


Hereby, we remark that $(C+1)^{-1}$ exists, since $(C+1)=\left(\frac{h \underline{d}}{2 \sqrt{4+h^{2} \underline{d}^{2}}}+\frac{1}{2}\right)=0$ is equivalent to $4+h^{2} \underline{d}^{2}=h^{2} \underline{d}^{2}$.

But, more important by eliminating $F_{2}^{+, 0}$ and $F_{1}^{+, 0}, F_{4}^{+, 0}$ we get the following conditions

$$
\left\{\begin{array}{c}
(C(C+1)-(A+B) B) F_{1}^{+, 1}+B F_{2}^{+, 1}=0, \\
B\left(F_{1}^{+, 1}-F_{4}^{+, 1}\right)+(C(C+1)-(A+B) B) F_{3}^{+, 1}=0 .
\end{array}\right.
$$

This leads to the following characterization for a function being a boundary value of a discrete monogenic function. For the proof we only remark that $C(C+1)-(A+B) B=$ $\frac{1}{2}\left(\frac{h \underline{d}}{\sqrt{4+h^{2} \underline{d}^{2}}}-1\right)$.

Theorem 4.2 Let $f \in l_{p}\left(\mathbb{Z}^{2}, \mathbb{C}_{3}\right)$ given by $f=f_{1}+e_{3}^{+} f_{2}+e_{3}^{-} f_{3}+e_{3}^{+} e_{3}^{-} f_{4}, f_{i} \in \mathbb{C}_{2}$. Then $f$ is the boundary value of a discrete monogenic function in the discrete upper half plane if and only if its $2 D$-Fourier transform $F=\mathcal{F}_{h} f$, with

$$
F(\underline{\xi})=F_{1}(\underline{\xi})+e_{3}^{+} F_{2}(\underline{\xi})+e_{3}^{-} F_{3}(\underline{\xi})+e_{3}^{+} e_{3}^{-} F_{4}(\underline{\xi}), \quad \underline{\xi} \in\left[-\frac{\pi}{h}, \frac{\pi}{h}\right]^{2},
$$

satisfies the system

$$
\left\{\begin{array}{c}
\frac{h \underline{d}-\sqrt{4+h^{2} \underline{d}^{2}}}{2} F_{1}+\frac{\widetilde{\widetilde{\xi}}}{\underline{d}} F_{2}=0, \\
\frac{h \underline{d}-\sqrt{4+h^{2} \underline{d}^{2}}}{2} F_{3}+\frac{\widetilde{\widetilde{\xi}}}{\underline{\underline{d}}}\left(F_{1}-F_{4}\right)=0 .
\end{array}\right.
$$

In this case the values on the layer $m_{3}=0$ are given by

$$
\left\{\begin{array}{c}
F_{2}^{+, 0}=\frac{\widetilde{\xi}_{-}}{\underline{d}}\left(\frac{h d}{2}+\sqrt{1+\left(\frac{h d}{2}\right)^{2}}\right) F_{1}, \\
F_{1}^{+, 0}-F_{4}^{+, 0}=\frac{\widetilde{\xi}_{-}}{\underline{d}}\left(\frac{h d}{2}+\sqrt{1+\left(\frac{h d}{2}\right)^{2}}\right) F_{3} .
\end{array}\right.
$$

and the discrete monogenic function itself by applying the discrete upper Cauchy transform.

The condition in the above theorem is given in terms of the component functions. But we would like to have it in terms of the function itself. To obtain such a condition we remark that we have

$$
\begin{aligned}
& e_{3}^{-} F=e_{3}^{-}\left(F_{1}-F_{4}\right)-\left(1+e_{3}^{+} e_{3}^{-}\right) F_{2}, \\
& e_{3}^{-} e_{3}^{+} F=-\left(1+e_{3}^{+} e_{3}^{-}\right) F_{1}-e_{3}^{-} F_{3} .
\end{aligned}
$$

Now, by abreviating $G=\frac{\widetilde{\xi}_{-}}{\underline{d}} \frac{h \underline{d}-\sqrt{4+h^{2} \underline{d}^{2}}}{2}$ in condition (4.42) in the above theorem we obtain $F_{1}=-G^{-1} F_{2}$ and $F_{3}=-G^{-1}\left(F_{1}-F_{4}\right)$. Hence, we have

$$
e_{3}^{-} e_{3}^{+} F=-G^{-1} e_{3}^{-}\left(F_{1}-F_{4}\right)+G^{-1}\left(1+e_{3}^{+} e_{3}^{-}\right) F_{2}=-G^{-1} e_{3}^{-} F=e_{3}^{-} G^{-1} F .
$$

Hence, we get

$$
e_{3}^{-}\left(e_{3}^{+} F-G^{-1} F\right)=0 .
$$

In the same way from $e_{3}^{+} F=e_{3}^{+} F_{1}+e_{3}^{+} e_{3}^{-} F_{3}$ and $e_{3}^{+} e_{3}^{-} F=e_{3}^{+} e_{3}^{-}\left(F_{1}-F_{4}\right)-e_{3}^{+} F_{2}$ together with condition (4.42) we get

$$
e_{3}^{+} e_{3}^{-} F=-G e_{3}^{+} e_{3}^{-} F_{3}-G e_{3}^{+} F_{1}=-G e_{3}^{+} F=e_{3}^{+} G F .
$$


Here, we arrive at

$$
e_{3}^{+}\left(e_{3}^{-} F-G F\right)=0 .
$$

Now, taking into account that $\left(e_{3}^{+} e_{3}^{-}+e_{3}^{-} e_{3}^{+}\right) F=-F$ we can sum up both terms and get

$$
\begin{aligned}
-F & =e_{3}^{-} G^{-1} F+e_{3}^{+} G F=e_{3}^{-} \frac{\tilde{\xi}_{-}}{\underline{d}} \frac{2}{h \underline{d}-\sqrt{4+h^{2} \underline{d}^{2}}} F+e_{3}^{+} \frac{\underline{\widetilde{\xi}}_{-}}{\underline{d}} \frac{h \underline{d}-\sqrt{4+h^{2} \underline{d}^{2}}}{2} F, \\
& =-\frac{\underline{\widetilde{\xi}}_{-}}{\underline{d}}\left(e_{3}^{-} \frac{2}{h \underline{d}-\sqrt{4+h^{2} \underline{d}^{2}}} F+e_{3}^{+} \frac{h \underline{d}-\sqrt{4+h^{2} \underline{d}^{2}}}{2}\right) F .
\end{aligned}
$$

This allows us to get the desired characterization in terms of the function instead in terms of its components.

Corollary 4.3 Let $f \in l_{p}\left(\mathbb{Z}^{2}, \mathbb{C}_{3}\right)$ be a boundary value of a discrete monogenic function in the upper half space. Then its $2 D$-Fourier transform $F=\mathcal{F}_{h} f$, satisfies the equation

$$
\frac{\tilde{\xi}_{-}}{\underline{d}}\left(e_{3}^{+} \frac{h \underline{d}-\sqrt{4+h^{2} \underline{d}^{2}}}{2}+e_{3}^{-} \frac{2}{h \underline{d}-\sqrt{4+h^{2} \underline{d}^{2}}}\right) F=F .
$$

Based on the above corollary we can introduce the operator

$$
H_{+} f=\mathcal{F}_{h}^{-1}\left[\frac{\widetilde{\xi}}{\underline{d}}\left(e_{3}^{+} \frac{h \underline{d}-\sqrt{4+h^{2} \underline{d}^{2}}}{2}+e_{3}^{-} \frac{2}{h \underline{d}-\sqrt{4+h^{2} \underline{d}^{2}}}\right)\right] \mathcal{F}_{h} f
$$

which satisfies $H_{+}^{2} f=f$.

In fact,

$$
\begin{aligned}
H_{+}^{2} f & =\frac{\widetilde{\xi}_{-}}{\underline{d}}\left(-e_{3}^{+} \frac{h \underline{d}-\sqrt{4+h^{2} \underline{d}^{2}}}{2}+e_{3}^{-} \frac{2}{h \underline{d}-\sqrt{4+h^{2} \underline{d}^{2}}}\right) \frac{\underline{\widetilde{\xi}}_{-}}{\underline{d}}\left(-e_{3}^{+} \frac{h \underline{d}-\sqrt{4+h^{2} \underline{d}^{2}}}{2}+e_{3}^{-} \frac{2}{h \underline{d}-\sqrt{4+h^{2} \underline{d}^{2}}}\right) f \\
& =-\left(-e_{3}^{+} \frac{h \underline{d}-\sqrt{4+h^{2} \underline{d}^{2}}}{2}+e_{3}^{-} \frac{2}{h \underline{d}-\sqrt{4+h^{2} \underline{d}^{2}}}\right)\left(-e_{3}^{+} \frac{h \underline{d}-\sqrt{4+h^{2} \underline{d}^{2}}}{2}+e_{3}^{-} \frac{2}{h \underline{d}-\sqrt{4+h^{2} \underline{d}^{2}}}\right) f \\
& =\left(e_{3}^{+} e_{3}^{-}+e_{3}^{-} e_{3}^{+}\right) f=-f .
\end{aligned}
$$

Now, let us take a look at the case of the lower half plane. For the layer $m_{3}=-1$ we can write (4.36) in terms of our abreviations:

$$
\left\{\begin{array}{ccc}
(1+C) F_{2}^{-,-1} & = & -B F_{1}^{-, 0}, \\
F_{1}^{-,-1}+(A+B) F_{2}^{-,-1} & = & -C F_{1}^{-, 0}, \\
F_{3}^{-,-1}+(A+B)\left(F_{1}^{-,-1}-F_{4}^{-,-1}\right) & = & -C F_{3}^{-, 0} \\
C\left(F_{1}^{-,-1}-F_{4}^{-,-1}\right)-(A+B) F_{2}^{-,-1}-F_{4}^{-,-1} & = & C F_{1}^{-, 0}-B F_{3}^{-, 0}
\end{array}\right.
$$

Here, we have

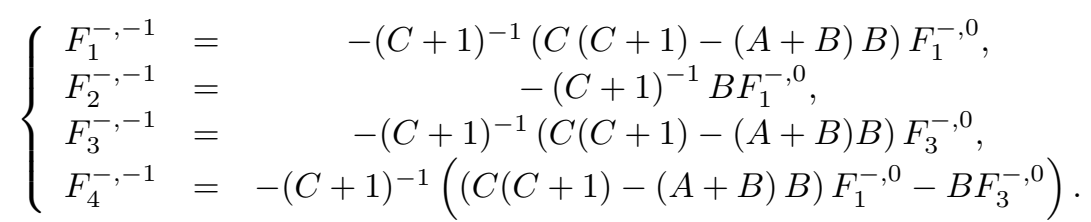


as well as the condition

$$
\left\{\begin{array}{c}
-B F_{1}^{-,-1}+(C(C+1)-(A+B) B) F_{2}^{-,-1}=0 \\
(C(C+1)-(A+B) B)\left(F_{1}^{-,-1}-F_{4}^{-,-1}\right)-B F_{3}^{-,-1}=0 .
\end{array}\right.
$$

In the same way as in the case of the discrete upper half space we arrive at the following theorem.

Theorem 4.4 Let $f \in l_{p}\left(\mathbb{Z}^{2}, \mathbb{C}_{3}\right)$ given by $f=f_{1}+e_{3}^{+} f_{2}+e_{3}^{-} f_{3}+e_{3}^{+} e_{3}^{-} f_{4}, f_{i} \in \mathbb{C}_{2}$. Then $f$ is the boundary value of a discrete monogenic function in the discrete lower half plane if and only if its $2 D$-Fourier transform $F=\mathcal{F}_{h} f$, with

$$
F(\underline{\xi})=F_{1}(\underline{\xi})+e_{3}^{+} F_{2}(\underline{\xi})+e_{3}^{-} F_{3}(\underline{\xi})+e_{3}^{+} e_{3}^{-} F_{4}(\underline{\xi}), \quad \underline{\xi} \in\left[-\frac{\pi}{h}, \frac{\pi}{h}\right]^{2},
$$

satisfies the system

$$
\left\{\begin{array}{c}
\frac{h \underline{d}-\sqrt{4+h^{2} \underline{d}^{2}}}{2} F_{2}-\frac{\widetilde{\xi}_{-}}{\underline{d}} F_{1}=0, \\
\frac{h \underline{d}-\sqrt{4+h^{2} \underline{d}^{2}}}{2}\left(F_{1}-F_{4}\right)-\frac{\widetilde{\xi}_{-}}{\underline{d}} F_{3}=0 .
\end{array}\right.
$$

The values on the layer $m_{3}=0$ can be obtained via

$$
\left\{\begin{array}{c}
F_{1}^{-, 0}=-\frac{\widetilde{\xi}_{-}}{\underline{d}}\left(\frac{h \underline{d}}{2}+\sqrt{1+\left(\frac{h d}{2}\right)^{2}}\right) F_{2}, \\
F_{3}^{-, 0}=-\frac{\widetilde{\xi}_{-}}{\underline{\underline{d}}}\left(\frac{h \underline{d}}{2}+\sqrt{1+\left(\frac{h d}{2}\right)^{2}}\right)\left(F_{1}-F_{4}\right)
\end{array}\right.
$$

and the discrete monogenic function itself by applying the discrete lower Cauchy transform.

Similar to the case of the discrete upper half plane we would like to have a characterization in terms of the function itself. Let us start again with

$$
\begin{aligned}
& e_{3}^{-} F=e_{3}^{-}\left(F_{1}-F_{4}\right)-\left(1+e_{3}^{+} e_{3}^{-}\right) F_{2}, \\
& e_{3}^{-} e_{3}^{+} F=-\left(1+e_{3}^{+} e_{3}^{-}\right) F_{1}-e_{3}^{-} F_{3} .
\end{aligned}
$$

Now, by abreviating $G=\frac{\widetilde{\xi}_{-}}{d} \frac{h \underline{d}-\sqrt{4+h^{2} \underline{d}^{2}}}{2}$ we can use condition (4.47) of the above theorem to obtain $F_{1}=G F_{2}$ and $F_{3}=G\left(F_{1}-F_{4}\right)$. This leads to

$$
e_{3}^{-} e_{3}^{+} F=G e_{3}^{-}\left(F_{1}-F_{4}\right)-G\left(1+e_{3}^{+} e_{3}^{-}\right) F_{2}=G e_{3}^{-} F=-e_{3}^{-} G F \text {. }
$$

Here, we get

$$
e_{3}^{-}\left(e_{3}^{+} F+G F\right)=0 .
$$

In the same way we start from $e_{3}^{+} F=e_{3}^{+} F_{1}+e_{3}^{+} e_{3}^{-} F_{3}$ and $e_{3}^{+} e_{3}^{-} F=e_{3}^{+} e_{3}^{-}\left(F_{1}-F_{4}\right)-e_{3}^{+} F_{2}$ and by combining it with condition (4.42) we obtain

$$
e_{3}^{+} e_{3}^{-} F=G^{-1} e_{3}^{+} e_{3}^{-} F_{3}+G^{-1} e_{3}^{+} F_{1}=G^{-1} e_{3}^{+} F=-e_{3}^{+} G^{-1} F .
$$

Hence, we have

$$
e_{3}^{+}\left(G^{-1} F+e_{3}^{-} F\right)=0 .
$$


Like in the case of the discrete upper half sphere we can again deduce

$$
\begin{aligned}
-F & =e_{3}^{-} e_{3}^{+} F+e_{3}^{+} e_{3}^{-} F=-e_{3}^{-} G F-e_{3}^{+} G^{-1} F \\
& =-e_{3}^{-} \frac{\widetilde{\xi}_{-}}{\underline{d}} \frac{h \underline{d}-\sqrt{4+h^{2} \underline{d}^{2}}}{2} F-e_{3}^{+} \frac{\underline{\widetilde{\xi}}_{-}}{\underline{d}} \frac{2}{h \underline{d}-\sqrt{4+h^{2} \underline{d}^{2}}} F \\
& =-\frac{\widetilde{\xi}_{-}}{\underline{d}}\left(-e_{3}^{-} \frac{h \underline{d}-\sqrt{4+h^{2} \underline{d}^{2}}}{2} F-e_{3}^{+} \frac{2}{h \underline{d}-\sqrt{4+h^{2} \underline{d}^{2}}}\right) F .
\end{aligned}
$$

This results in the following corollary.

Corollary 4.5 Let $f \in l_{p}\left(\mathbb{Z}^{2}, \mathbb{C}_{3}\right)$ be a boundary value of a discrete monogenic function in the lower half space. Then its $2 D$-Fourier transform $F=\mathcal{F}_{h} f$, satisfies the equation

$$
-\frac{\underline{\widetilde{\xi}}_{-}}{\underline{d}}\left(e_{3}^{+} \frac{2}{h \underline{d}-\sqrt{4+h^{2} \underline{d}^{2}}}+e_{3}^{-} \frac{h \underline{d}-\sqrt{4+h^{2} \underline{d}^{2}}}{2}\right) F=F .
$$

Here, we can again point out that the above equation induces the following operator

$$
H_{-} f=-\mathcal{F}_{h}^{-1}\left[\frac{\tilde{\xi}}{\underline{d}}\left(e_{3}^{+} \frac{2}{h \underline{d}-\sqrt{4+h^{2} \underline{d}^{2}}}+e_{3}^{-} \frac{h \underline{d}-\sqrt{4-h^{2} \underline{d}^{2}}}{2}\right)\right] \mathcal{F}_{h} f
$$

which satisfies $H_{-}^{2} f=f$.

In fact,

$$
\begin{aligned}
H_{-}^{2} f & =\frac{\widetilde{\xi}_{-}}{\underline{d}}\left(e_{3}^{+} \frac{2}{h \underline{d}-\sqrt{4+h^{2} \underline{d}^{2}}}-e_{3}^{-} \frac{h \underline{d}-\sqrt{4-h^{2} \underline{d}^{2}}}{2}\right) \frac{\widetilde{\xi}_{-}}{\underline{d}}\left(e_{3}^{+} \frac{2}{h \underline{d}-\sqrt{4+h^{2} \underline{d}^{2}}}-e_{3}^{-} \frac{h \underline{d}-\sqrt{4-h^{2} \underline{d}^{2}}}{2}\right) f \\
& =-\left(e_{3}^{+} \frac{2}{h \underline{d}-\sqrt{4+h^{2} \underline{d}^{2}}}-e_{3}^{-} \frac{h \underline{d}-\sqrt{4-h^{2} \underline{d}^{2}}}{2}\right)\left(e_{3}^{+} \frac{2}{h \underline{d}-\sqrt{4+h^{2} \underline{d}^{2}}}-e_{3}^{-} \frac{h \underline{d}-\sqrt{4-h^{2} \underline{d}^{2}}}{2}\right) f \\
& =\left(e_{3}^{-} e_{3}^{+}+e_{3}^{+} e_{3}^{-}\right) f=-f .
\end{aligned}
$$

Furthermore, we would like to point out that conditions (4.43) and condition (4.47) are linear which motivates the following definition.

Definition 4.6 We define the upper discrete Hardy space $h_{p}^{+}$as the space of discrete functions $f \in l_{p}\left(\mathbb{Z}^{2}, \mathbb{C}_{3}\right)$ which the discrete $2 D$-Fourier transform fulfil system (4.43) and the lower discrete Hardy space $h_{p}^{+}$as the space of discrete functions $f \in l_{p}\left(\mathbb{Z}^{2}, \mathbb{C}_{3}\right)$ which the discrete 2D-Fourier transform fulfil system (4.47).

First of all it is obvious from the construction that the upper and lower discrete Hardy spaces are indeed spaces. The principal question at this point is: Does the decomposition of the continuous $L_{p}$-space into Hardy spaces still hold true in the discrete case?

We start by studying the intersection of the two spaces, i.e. $h_{p}^{+} \cap h_{p}^{-}$. This means we are interested in what functions $f$ have their discrete 2D-Fourier transform $F=\mathcal{F}_{h} f$ fulfil (4.41) 
and (4.46), i.e.

$$
\left\{\begin{array}{c}
(C(C+1)-(A+B) B) F_{1}+B F_{2}=0 \\
B\left(F_{1}-F_{4}\right)+(C(C+1)-(A+B) B) F_{3}=0 \\
-B F_{1}+(C(C+1)-(A+B) B) F_{2}=0 \\
(C(C+1)-(A+B) B)\left(F_{1}-F_{4}\right)-B F_{3}=0
\end{array}\right.
$$

From the first and third equation we get

$$
\left(B^{2}+(C(C+1)-(A+B) B)^{2}\right) F_{2}=0
$$

Here, $B^{2}+(C(C+1)-(A+B) B)^{2} \neq 0$ since $h^{2} \underline{d}^{2}=(h \underline{d}-2) \sqrt{4+h^{2} \underline{d}^{2}}$ does not have any positive roots. This implies $F_{2}=F_{1}=0$. Now, from the second and fourth equation we obtain

$$
\left(B^{2}+(C(C+1)-(A+B) B)^{2}\right) F_{3}=0
$$

which again implies that $F_{3}=F_{4}=0$.

Due to the properties of $H_{+}$and $H_{-}$we can introduce the projectors $P_{+}=\frac{1}{2}\left(I+H_{+}\right)$ and $P_{-}=\frac{1}{2}\left(I+H_{-}\right)$. From the above investigations it is clear that $f \in h_{p}^{+}$is equivalent to $P_{+} f=f$ and $f \in h_{p}^{-}$means $P_{-} f=f$.

The question is now, what is $P_{+} f+P_{-} f$ ? Here we have:

$$
\begin{aligned}
& \mathcal{F}_{h}\left[P_{+} f+P_{-} f\right] \\
& =\frac{1}{2} \mathcal{F}_{h} f+\frac{1}{2}\left[\frac{\underline{\xi}}{\underline{d}}\left(e_{3}^{+} \frac{h \underline{d}-\sqrt{4+h^{2} \underline{d}^{2}}}{2}+e_{3}^{-} \frac{2}{h \underline{d}-\sqrt{4+h^{2} \underline{d}^{2}}}\right)\right] \mathcal{F}_{h} f \\
& +\frac{1}{2} \mathcal{F}_{h} f-\frac{1}{2}\left[\frac{\underline{\tilde{\xi}}}{\underline{d}}\left(e_{3}^{+} \frac{2}{h \underline{d}-\sqrt{4+h^{2} \underline{d}^{2}}}+e_{3}^{-} \frac{h \underline{d}-\sqrt{4-h^{2} \underline{d}^{2}}}{2}\right)\right] \mathcal{F}_{h} f \\
& =\mathcal{F}_{h} f+\frac{1}{2} \frac{\widetilde{\widetilde{\xi}}_{-}}{\underline{d}}\left(\frac{2}{h \underline{d}-\sqrt{4+h^{2} \underline{d}^{2}}}-\frac{h \underline{d}-\sqrt{4+h^{2} \underline{d}^{2}}}{2}\right)\left(e_{3}^{+}-e_{3}^{-}\right) \mathcal{F}_{h} f \\
& =\mathcal{F}_{h} f+\frac{h \underline{d}}{2} \frac{\tilde{\xi}}{\underline{d}}\left(e_{3}^{+}-e_{3}^{-}\right) \mathcal{F}_{h} f .
\end{aligned}
$$

Therefore, we get

$$
P_{+} f+P_{-} f=f+\frac{h}{2} \mathcal{F}_{h}^{-1}\left[\widetilde{\widetilde{\xi}}_{-} \mathcal{F}_{h} f\right] .
$$

The last equation can be rewritten as

$$
P_{+} f+P_{-} f=f+\frac{h}{2} \sum_{k=1}^{2}\left[e_{k}^{+} \partial_{h}^{-k}+e_{k}^{-} \partial_{h}^{+k}\right] f .
$$

\section{Convergence results}

Finally, we are interested how far our discrete results correspond to the results in the continuous case. That means we need to study what happens if our lattice constant $h$ goes to zero. Let us denote by $C_{\Gamma}$ the continuous Cauchy transform

$$
C_{\Gamma} f(y)=\int_{\mathbb{R}^{2}} E(x-y)\left(-e_{3}\right) f(x) d \Gamma_{x}, \quad y=\left(\underline{y}, y_{3}\right) \in \mathbb{R}^{3}, y_{3}>0,
$$


with $E(x)$ being the fundamental solution to the Dirac operator $D=\sum_{j=1}^{3} e_{j} \frac{\partial}{\partial_{x_{j}}}$ (c.f. [10]). Then we can state the following theorem.

Theorem 5.1 If $f \in \mathbb{L}_{p}\left(\mathbb{R}^{2}, \mathbb{C}_{3}\right) \cap C^{\alpha}\left(G, \mathbb{C}_{3}\right), 0<\alpha \leqslant 1,1 \leqslant p<+\infty$, then we get

$$
\left|C_{\Gamma} f(y)-C^{+} f(y)\right| \leqslant C h^{2},
$$

for any point $y \in \mathbb{Z}_{+}$and $C>0$ being a constant independent on $h$.

Proof: Let us first remark that for functions $f \in \mathbb{L}_{p}\left(\mathbb{R}^{2}, \mathbb{C}_{3}\right) \cap C^{\alpha}\left(G, \mathbb{C}_{3}\right), 0<\alpha \leqslant 1,1<p<\infty$, we have $\|f(\cdot h)\|_{p+2 / \alpha} \leqslant C\|f\|_{L_{p}}$. This is an easy adaptation of Lemma 3.1 in [6]. We will consider a fixed point $y$ such that there exist for a given $h$ an $m_{h}$ with $y=m_{h} h$ and $y_{3}>1$. Now, let $W(x)$ be a square with center $x$ and edge length $h$. Furthermore, let $1 / q+1 / p=1$ and $1 / s+1 /(p+2 / \alpha)=1$ Then we have

$$
\begin{aligned}
& \left|C_{\Gamma} f(y)-C^{+} f(y)\right| \\
& =\mid \int_{\mathbb{R}^{2}} E\left(x-\underline{y},-y_{3}\right)\left(-e_{3}\right) f(x, 0) d \Gamma_{x}+\sum_{\underline{n} \in \mathbb{Z}^{2}}\left[E_{h}^{-+}\left(\underline{n} h-\underline{y},-y_{3}\right) e_{3}^{+} f(\underline{n} h, 1 h)\right. \\
& \left.+E_{h}^{-+}\left(\underline{n} h-\underline{y}, h-y_{3}\right) e_{3}^{-} f(\underline{n} h, 0)\right] h^{2} \\
& \leqslant \mid \sum_{\underline{n} \in \mathbb{Z}^{2}}\left(\int_{W(\underline{n} h)} E\left(x-\underline{y},-y_{3} h\right)\left(-e_{3}^{+}-e_{3}^{-}\right) f(x, 0) d \Gamma_{x}\right. \\
& \left.+\left[E_{h}^{-+}\left(\underline{n} h-\underline{y},-y_{3} h\right) e_{3}^{+} f(\underline{n} h, 1 h)+E_{h}^{-+}\left(\underline{n} h-\underline{y}, h-y_{3}\right) e_{3}^{-} f(\underline{n} h, 0)\right]\right) h^{2} \mid \\
& \leqslant \sum_{\underline{n} \in \mathbb{Z}^{2}}\left(\left|E_{h}^{-+}\left(\underline{n} h-\underline{y},-y_{3}\right)-E\left(\underline{n} h-\underline{y},-y_{3}\right) e_{3}^{+} f(\underline{n} h, 1 h)\right| h^{2}\right. \\
& +\left|E_{h}^{-+}\left(\underline{n} h-\underline{y}, h-y_{3}\right)-E\left(\underline{n} h-\underline{y}, h-y_{3}\right) e_{3}^{-} f(\underline{n} h, 0)\right| h^{2} \\
& +\left|E\left(\underline{n} h-\underline{y},-y_{3}\right) e_{3}^{+} f(\underline{n} h, 1 h) h^{2}-\int_{W(\underline{n} h)} E\left(x-\underline{y},-y_{3}\right) e_{3}^{+} f(x, 0) d \Gamma_{x}\right| \\
& \left.+\left|E\left(\underline{n} h-\underline{y}, h-y_{3}\right) e_{3}^{-} f(\underline{n} h, 0) h^{2}-\int_{W(\underline{n} h)} E\left(x-\underline{y},-y_{3}\right) e_{3}^{-} f(x, 0) d \Gamma_{x}\right|\right) \\
& \leqslant c_{1}^{2} \sum_{\underline{n} \in \mathbb{Z}^{2}}\left(\left|E_{h}^{-+}\left(\underline{n} h-\underline{y},-y_{3}\right)-E\left(\underline{n} h-\underline{y},-y_{3}\right)\right||f(\underline{n} h, 1 h)| h^{2}\right. \\
& +\left|E_{h}^{-+}\left(\underline{n} h-\underline{y}, h-y_{3}\right)-E(\underline{n} h-\underline{y}, h-y)\right||f(\underline{n} h, 0)| h^{2} . \\
& +\left|E\left(\underline{n} h-\underline{y},-y_{3}\right) e_{3}^{+} f(\underline{n} h, 1 h) h^{2}-\int_{W(\underline{n} h)} E\left(x-\underline{y},-y_{3}\right) e_{3}^{+} f(x, 0) d \Gamma_{x}\right| \\
& \left.+\left|E\left(\underline{n} h-\underline{y}, h-y_{3}\right) e_{3}^{-} f(\underline{n} h, 0) h^{2}-\int_{W(\underline{n} h)} E\left(x-\underline{y},-y_{3} h\right) e_{3}^{-} f(x, 0) d \Gamma_{x}\right|\right)
\end{aligned}
$$


with $c_{1}=2^{3 / 2}$. Now, Hölder's inequality and Lemma 2.3 provides

$$
\begin{aligned}
& \sum_{\underline{n} \in \mathbb{Z}^{2}}\left|E_{h}^{-+}\left(\underline{n} h-\underline{y},-y_{3}\right)-E\left(\underline{n} h-\underline{y},-y_{3}\right)\right||f(\underline{n} h, 1 h)| h^{2} \\
& \leqslant\left(\sum_{\underline{n} \in \mathbb{Z}^{2}}\left|E_{h}^{-+}\left(\underline{n} h-\underline{y},-y_{3}\right)-E\left(\underline{n} h-\underline{y},-y_{3}\right)\right|^{s} h^{2}\right)^{1 / s}\|f\|_{p+2 / \alpha} \\
& \leqslant C_{1} h^{1+2 / s}\|f\|_{p+2 / \alpha}
\end{aligned}
$$

as well as

$$
\begin{aligned}
& \sum_{\underline{n} \in \mathbb{Z}^{2}}\left|E_{h}^{-+}\left(\underline{n} h-\underline{y}, h-y_{3}\right)-E\left(\underline{n} h-\underline{y}, h-y_{3}\right)\right||f(\underline{n} h, 0)| h^{2} \\
& \leqslant\left(\sum_{\underline{n} \in \mathbb{Z}^{2}}\left|E_{h}^{-+}\left(\underline{n} h-\underline{y}, h-y_{3}\right)-E\left(\underline{n} h-\underline{y}, h-y_{3}\right)\right|^{s} h^{3}\right)^{1 / s}\|f\|_{p+2 / \alpha} \\
& \leqslant C_{1} h^{1+2 / s}\|f\|_{p+2 / \alpha}
\end{aligned}
$$

The term

$$
\left|E\left(\underline{n} h-\underline{y},-y_{3}\right) e_{3}^{+} f(\underline{n} h, 1 h) h^{2}-\int_{W(\underline{n})} E\left(x-\underline{y},-y_{3}\right) e_{3}^{+} f(x, 0) d \Gamma_{x}\right|
$$

can be estimated by

$$
\begin{aligned}
\leqslant & \mid \int_{W(\underline{n})} E\left(\underline{n} h-\underline{y},-y_{3}\right) e_{3}^{+}[f(\underline{n} h, 1 h)-f(x, 0)] d \Gamma_{x} \\
& +\int_{W(\underline{n})}\left[E\left(\underline{n} h-\underline{y},-y_{3}\right)-E\left(x-\underline{y},-y_{3}\right)\right] e_{3}^{+} f(x, 0) d \Gamma_{x} \mid
\end{aligned}
$$

We can estimate the first term using Hölders inequality:

$$
\begin{aligned}
& \left|\int_{W(\underline{n})} E\left(\underline{n} h-\underline{y},-y_{3}\right) e_{3}^{+}[f(\underline{n} h, 1 h)-f(x, 0)] d \Gamma_{x}\right| \\
& \leqslant c_{1}\left(\int_{W(\underline{n})}\left|E\left(\underline{n} h-\underline{y},-y_{3}\right)\right|^{q} d \Gamma_{x}\right)^{1 / q}\left(\int_{W(\underline{n})}|f(\underline{n} h, 1 h)-f(x, 0)|^{p} d \Gamma_{x}\right)^{1 / p} \\
& \leqslant C_{2} \frac{1}{\left|\left(\underline{n} h-\underline{y},-y_{3}\right)\right|^{2}} h^{2}
\end{aligned}
$$

For the second term we can use the Taylor expansion for the kernel:

$$
\begin{aligned}
& \left|\int_{W(\underline{n})}\left[E\left(\underline{n} h-\underline{y},-y_{3}\right)-E\left(x-\underline{y},-y_{3}\right)\right] e_{3}^{+} f(x, 0) d \Gamma_{x}\right| \\
& \leqslant c_{1} \int_{W(\underline{n})} \sum_{k=1}^{2}\left|\partial_{x_{k}} E\left(\underline{n} h-\underline{x},-y_{3}\right)\right|_{x=\underline{y}}|| x_{k}-n_{k} h|| f(x, 0) \mid d \Gamma_{x} \\
& \leqslant C_{3} \frac{h^{2+2 / p}}{\left.\mid \underline{n} h-y,-y_{3}\right)\left.\right|^{3}}\left(\int_{W(n)} \mid f\left(x,\left.0\right|^{q} d \Gamma_{x}\right)^{1 / q}\right.
\end{aligned}
$$


It follows the result.

Since we have the convergence of our discrete Cauchy transform to the continuous Cauchy transform the question remains how far (4.35) and (4.36) correspond to the classic continuous case when $h \rightarrow 0$.

When $h \rightarrow 0$ on both sides of the terms (4.35) and (4.36), we get

$$
\begin{aligned}
& \left(\frac{1}{2} \frac{-i \underline{\xi}}{|\underline{\xi}|}-\frac{1}{2} e_{3}^{+}\right) e_{3}^{-} \widehat{f}^{+}(\underline{\xi}, 0)+\left(\frac{1}{2} \frac{-i \underline{\xi}}{|\underline{\xi}|}-\frac{1}{2} e_{3}^{-}\right) e_{3}^{+} \widehat{f}^{+}(\underline{\xi}, 0)=0, \\
& \left(\frac{1}{2} \frac{-i \underline{\xi}}{|\underline{\xi}|}+\frac{1}{2} e_{3}^{-}\right) e_{3}^{+} \widehat{f}^{+}(\underline{\xi}, 0)+\left(\frac{1}{2} \frac{-i \underline{\xi}}{|\underline{\xi}|}+\frac{1}{2} e_{3}^{+}\right) e_{3}^{-} \widehat{f}^{+}(\underline{\xi}, 0)=-\widehat{f}^{+}(\underline{\xi}, 0),
\end{aligned}
$$

as well as

$$
\begin{aligned}
& \left(\frac{1}{2} \frac{-i \underline{\xi}}{|\underline{\xi}|}+\frac{1}{2} e_{3}^{-}\right) e_{3}^{+} \widehat{f}^{-}(\underline{\xi}, 0)+\left(\frac{1}{2} \frac{-i \underline{\xi}}{|\underline{\xi}|}+\frac{1}{2} e_{3}^{+}\right) e_{3}^{-\widehat{f}^{-}}(\underline{\xi}, 0)=0, \\
& \left(\frac{1}{2} \frac{-i \underline{\xi}}{|\underline{\xi}|}-\frac{1}{2} e_{3}^{+}\right) e_{3}^{-\widehat{f}^{-}}(\underline{\xi}, 0)+\left(\frac{1}{2} \frac{-i \underline{\xi}}{|\underline{\xi}|}-\frac{1}{2} e_{3}^{-}\right) e_{3}^{+} \widehat{f}^{-}(\underline{\xi}, 0)=\widehat{f}^{-}(\underline{\xi}, 0),
\end{aligned}
$$

herby $\widehat{f}^{ \pm}(\underline{\xi}, 0)$ denote the limits of $F^{ \pm, \pm 1}(\underline{\xi})=\mathcal{F}_{h} f^{ \pm}(\underline{\xi}, \pm 1)$, that is to say, $F^{ \pm, 0}(\underline{\xi})=\mathcal{F}_{h} f^{ \pm}(\underline{\xi}, 0)$, when $h \rightarrow 0$, respectively. Therefore, we obtain

$$
\begin{aligned}
& \frac{1}{2} \frac{-i \underline{\xi}}{|\underline{\xi}|}\left(e_{3}^{-}+e_{3}^{+}\right) \hat{f}^{+}(\underline{\xi}, 0)=\frac{1}{2}\left(e_{3}^{+} e_{3}^{-}+e_{3}^{-} e_{3}^{+}\right) \hat{f}^{+}(\underline{\xi}, 0), \\
& \frac{1}{2} \frac{-i \underline{\xi}}{|\underline{\xi}|}\left(e_{3}^{-}+e_{3}^{+}\right) \hat{f}^{+}(\underline{\xi}, 0)=-\widehat{f}^{+}(\underline{\xi}, 0)-\frac{1}{2}\left(e_{3}^{+} e_{3}^{-}+e_{3}^{-} e_{3}^{+}\right) \widehat{f}^{+}(\underline{\xi}, 0),
\end{aligned}
$$

as well as

$$
\begin{aligned}
& \frac{1}{2} \frac{-i \underline{\xi}}{|\underline{\xi}|}\left(e_{3}^{-}+e_{3}^{+}\right) \widehat{f}^{-}(\underline{\xi}, 0)=-\frac{1}{2}\left(e_{3}^{+} e_{3}^{-}+e_{3}^{-} e_{3}^{+}\right) \widehat{f}^{-}(\underline{\xi}, 0) \\
& \frac{1}{2} \frac{-i \underline{\xi}}{|\underline{\xi}|^{2}}\left(e_{3}^{-}+e_{3}^{+}\right) \widehat{f}^{-}(\underline{\xi}, 0)=\widehat{f}^{-}(\underline{\xi}, 0)+\frac{1}{2}\left(e_{3}^{+} e_{3}^{-}+e_{3}^{-} e_{3}^{+}\right) \widehat{f}^{-}(\underline{\xi}, 0) .
\end{aligned}
$$

Now, if we look at our splitting of the Euclidean basis $e_{3}=e_{3}^{-}+e_{3}^{+}$with $e_{3}^{+} e_{3}^{-}+e_{3}^{-} e_{3}^{+}=-1$, equations (5.54) and (5.55), respectively, can be rewritten as

$$
\begin{aligned}
& \frac{-i \underline{\xi}}{|\underline{\xi}|}\left(-e_{3}\right) \widehat{f}^{+}(\underline{\xi}, 0)=\widehat{f}^{+}(\underline{\xi}, 0), \\
& \frac{-i \underline{\xi}}{|\underline{\xi}|} e_{3} \widehat{f}^{-}(\underline{\xi}, 0)=\widehat{f}^{-}(\underline{\xi}, 0),
\end{aligned}
$$

which corresponds exactly to the Plemelj formulae on the half space in the continuous case [16].

\section{References}

[1] F. Brackx, H. De Schepper, F. Sommen, L. Van de Voorde, Discrete Clifford analysis: a germ of function theory. In: I. Sabadini, M. Shapiro, F. Sommen (eds.), Hypercomplex Analysis, Birkhäuser, 2009, 37-53. 
[2] P. Cerejeiras, N. Faustino, N. Vieira, Numerical Clifford analysis for nonlinear Schrödinger problem, Numerical Methods for Partial Differential Equations 24(4) (2008), 1181-1202.

[3] Delanghe R., Sommen F., Soucek V., Clifford Algebra and Spinor-Valued Functions, Kluwer Academic, Dordrecht, 1992.

[4] H. De Ridder, H. De Schepper, U. Kähler, F. Sommen, Discrete function theory based on skew Weyl relations, Proceeding of the American Mathematical Society 138(9) (2010), 3241-3256.

[5] Hommel, A., Fundamentallösungen partieller Differentialoperatoren und die Lösung diskreter Randwertprobleme mit Hilfe von Differenzenpotentialen. PhD thesis, BauhausUniversitaät Weimar, Germany, 1998.

[6] N. Faustino, K. Gürlebeck, A. Hommel, U. Kähler, Difference potentials for the NavierStokes equations in unbounded domains, J. Diff. Eq. E Appl. 12(6) (2006), 577-595.

[7] N. Faustino, U. Kähler, F. Sommen, Discrete Dirac operators in Clifford analysis, Adv. Appl. Cliff. Alg. 17(3) (2007), 451-467.

[8] G.B. Folland, E.M. Stein, Hardy spaces on homogeneous groups, Princeton University Press, 1982

[9] E. Forgy, U. Schreiber, Discrete differential geometry on causal graphs, preprint (2004), arXiv:math-ph/0407005v1.

[10] Gürlebeck, K. and W. Sprößig, Quaternionic and Clifford calculus for physicists and engineers, Wiley, 1997.

[11] Gürlebeck, K. and and Hommel A., On finite difference Dirac operators and their fundamental solutions, Adv. Appl. Cliff. Alg. 11(S2) (2001), 89-106.

[12] I. Kanamori, N. Kawamoto, Dirac-Kaehler Fermion from Clifford Product with Noncommutative Differential Form on a Lattice, Int.J.Mod.Phys. A19 (2004), 695-736 .

[13] E.M. Stein, G. Weiss, Introduction to Fourier Analysis on Euclidean Spaces, Princeton University Press, 1971.

[14] C. Li, A. McIntosh, and T. Qian, Clifford algebras, Fourier transforms, and singular convolution operators on Lipschitz surfaces, Revista Matemática Iberoamericana, 10 (1994), 665-721.

[15] Lovasz, L., Discrete Analytic Functions: An Exposition, Surveys in differential geometry, Vol. IX (2004), 1-44.

[16] A. McIntosh, Clifford Algebras, Fourier theory, singular integrals, and harmonic functions on Lipschitz domains, in Clifford Algebras in Analysis and related Topics, edt. by J. Ryan, CRC Press, Boca Raton, 1996, 33-88.

[17] V.S. Ryabenskij, The method of difference potentials for some problems of continuum mechanics, Nauka, Moscow, 1984 (Russian).

[18] J. Vaz, Clifford-like calculus over lattices, Adv. Appl. Cliff. Alg. 7(1) (1997), 37-70. 